Review

\title{
The emerging role of Notch pathway in ageing: Focus on the related mechanisms in age-related diseases
}

\author{
Carmela Rita Balistreri ${ }^{\mathrm{a}, *}$, Rosalinda Madonna ${ }^{\mathrm{b}}$, Gerry Melino $^{\mathrm{c}, \mathrm{d}}$, Calogero Caruso $^{\mathrm{a}}$ \\ a Department of Pathobiology and Medical Biotechnologies, University of Palermo, 90134 Palermo, Italy \\ b Center of Excellence on Aging, Institute of Cardiology, Department of Neurosciences, Imaging and Clinical Sciences “G. d'Annunzio" University, 66100 \\ Chieti, Italy \\ ${ }^{c}$ Medical Research Council Toxicology Unit, Leicester LE1 9HN, UK \\ d University of Rome Tor Vergata, 00133 Rome, Italy
}

\section{A R T I C L E I N F O}

\section{Article history:}

Received 21 March 2016

Received in revised form 10 June 2016

Accepted 16 June 2016

Available online 17 June 2016

\section{Keywords:}

Notch pathway

Ageing

Age-related diseases

a signaling complex network

Involved mechanisms

Biomarkers and targets for personalized

treatments

\begin{abstract}
A B S T R A C T
Notch signaling is an evolutionarily conserved pathway, which is fundamental for the development of all tissues, organs and systems of human body. Recently, a considerable and still growing number of studies have highlighted the contribution of Notch signaling in various pathological processes of the adult life, such as age-related diseases. In particular, the Notch pathway has emerged as major player in the maintenance of tissue specific homeostasis, through the control of proliferation, migration, phenotypes and functions of tissue cells, as well as in the cross-talk between inflammatory cells and the innate immune system, and in onset of inflammatory age-related diseases. However, until now there is a confounding evidence about the related mechanisms. Here, we discuss mechanisms through which Notch signaling acts in a very complex network of pathways, where it seems to have the crucial role of hub. Thus, we stress the possibility to use Notch pathway, the related molecules and pathways constituting this network, both as innovative (predictive, diagnostic and prognostic) biomarkers and targets for personalised treatments for age-related diseases.
\end{abstract}

(c) 2016 Elsevier B.V. All rights reserved.

\section{Contents}

1. Introduction

.50

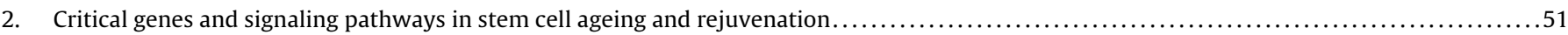

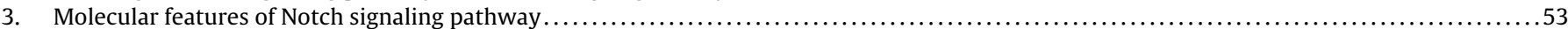

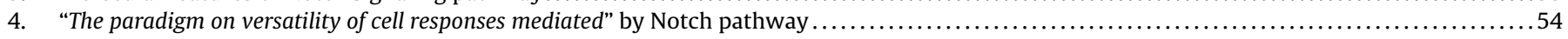

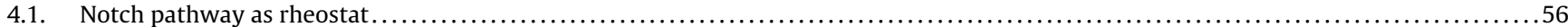

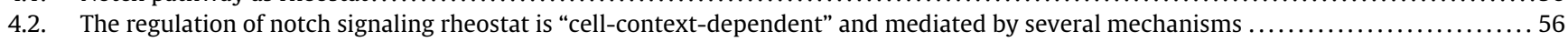

5. Ageing as the key driver of both age-related inflammation and the development of age-related diseases: focus on a large complex network of

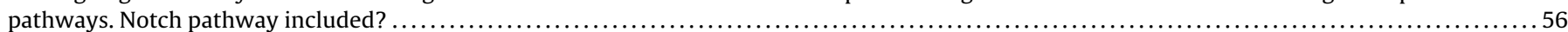

5.1. Notch in monocyte/macrophage cells, activation and functions: its cooperation with TLR and NF-kB pathways ....................57

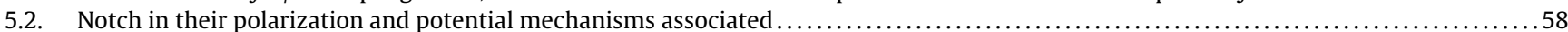

6. Notch pathway in context of cardiovascular inflammatory conditions related to onset of CVDs, and other age-related diseases................59

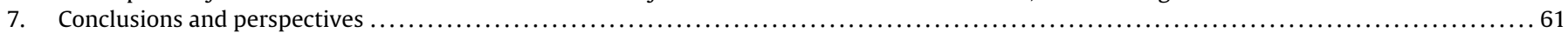

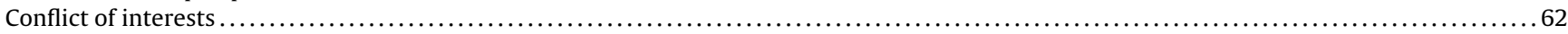

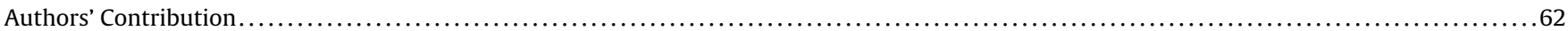

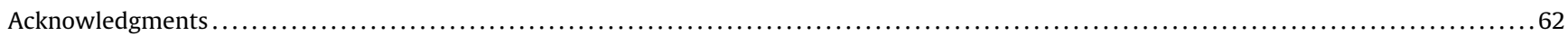

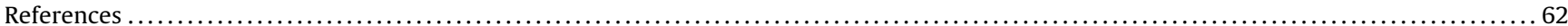

\section{Introduction}

\footnotetext{
* Corresponding author.

E-mail address: carmelarita.balistreri@unipa.it (C.R. Balistreri).
}

Cardiovascular system, extensively distributed among cells, tissues, organs, apparatuses and systems of the human body, has the fundamental role to supply oxygen and nutrients and brings 
away metabolic wastes. In addition, it also has capacity to sustain tissue growth and repair, and to provide cells and molecules for inducing immune surveillance. Alterations in genesis, homeostasis, structure and function of cardiovascular system determine, with advancing age, the onset and the progression of several agerelated diseases. For examples, abnormalities in cardiovascular self repair/regeneration and growth maintenance may exacerbate ischemic conditions, such as stroke and myocardial infarction (MI). Excessive angiogenesis, on the one hand, and abnormal vascular endothelium damage, on the other, may, while, induce cancer, inflammation, atherosclerosis, thrombosis, hypertension, diabetes and neurodegenerative disorders, such as Alzheimer disease (AD). This evidence has drawn attention of entire scientific community for searching urgent interventions, both in preventive measures and biomedicine research, in order to decrease the growing incidence of these diseases associated with "old population phenomenon". On the other hand, the most important determinant of cardiovascular health is person's age, and precisely the person biological age, as recently suggested in our studies (Balistreri et al., 2012 , 2014). By 2030, approximately $20 \%$ of the population will be aged 65 or older (Edwards, 2012). In this age group, age-related diseases, and particularly cardiovascular diseases (CVDs), will result in $40 \%$ of all deaths and rank as the leading cause (Heidenreich et al., 2011). Accordingly, in the last years the study of "endothelium dysfunction" and the related cellular and molecular mechanisms has obtained the major interest in a large number of research groups. This is in accordance to well recognized evidence that endothelium dysfunction is the first pathological condition associated not only with all CVDs, but also with other age-related diseases, being the endothelial cells (ECs) components of the stroma of all tissues and organs. Accumulation of EC damages followed by death and impaired cardiovascular self repair, due principally to altered agerelated function of tissue-specific resident and circulating stem or progenitor cells, characterize the onset of endothelial dysfunction (Regina et al., 2016). Precisely, when an imbalance between damage/death and regeneration of the endothelium occurs, integrity and function of endothelium is threatened, and the origin and progression of CVDs, or other age-related diseases, is favored (Madonna et al., 2016a, 2016b).

Experimental studies on endothelium have led to relevant and promising discoveries and advances. Among these findings, strong opinion has been emerging that the solution, to counteract the onset of age-related diseases, has the roots in the cellular mechanisms and evolutionally conserved signaling pathways of the cells, as the stem and progenitor cells (i.e. hematopoietic and no-hematopoietic stem cells and their progenitors), involved in the genesis of vessels, "vasculargenesis", tissues, organs and systems. This is in accordance to the innovative concepts of fetal programming-imprinting based on theory popularized, for the first time in 1989, by Dr David Barker, and defined as "the fetal origin of adult disease (FOAD)" (Barker et al., 1989, 2009; Barker, 2004) (see Box 1). Such concepts have been corroborated by valid data obtained from diverse human cohorts and various animal models, leading to other emerging speculations as the concepts of fetal vascular ageing proposed in 2011 by Pitale and Sahasrabuddhe (2011). Thus, David Barker's observations continue to be confirmed and tested in the time across multicultural populations and animal models subjected to various constraints (Barker et al., 1989, 2009; Barker, 2004; Calkins and Devaskar, 2011). Indeed, the important implications of FOAD hypothesis has been also underlined by the following statement of World Health Organization, "The global burden of death, disability and loss of human capital as a result of impaired fetal development is huge and affects both developed and developing countries (http://www.who.int/int/nutrition/topics/fetal_dev)".

Based on these observations, the field of stem cell biology and evolutionally conserved signaling pathways is becoming the gold

\section{Box 1: From the theory of Dr David Barker defined as "the fetal origin of adult disease (FOAD)" to concepts of fetal programming-imprinting.}

The FOAD hypothesis holds that events during early development have a profound impact on one's risk for development of future adult disease Barker et al., 1989, 2009; Barker, 2004; Calkins and Devaskar, 2011). Precisely, it sustains "the fetal programming concept". The notion of fetal programming implies that, during critical period of prenatal growth, a complex interplay in genetic composition, intrauterine conditions and epigenetic transmission between maternal milieu and fetus evocated by hormonal (i.e. hormonal status may be modified and exacerbated by assisted reproductive technologies, as recently demonstrated by Padhee and colleagues study; Padhee et al., 2015) and nutrient maternal changes may alter the full expression of fetal genome leading to permanent effects on large range of physical conditions, characterizing the modified and permanent phenotype of fetus. For example, low birth weight, a surrogate marker of poor fetal growth and nutrition, is linked to coronary artery disease, hypertension, obesity, and insulin resistance. Clues originally arose from large 20th century, European birth registries. Today, large, diverse human cohorts and various animal models have extensively replicated these original observations.

object of research of a large number of studies. Accordingly, it is emerging the fundamental role of an evolutionally conserved signaling pathway, the Notch pathway, in stem/progenitor cell fate decision, as well as in the life cycle of adult cells (Fiúza and Arias, 2007). Notch signaling pathway, by itself or cross-talking with other pathways (see below), has been shown to have a large range of critical functions during the development not only of cardiovascular system, but also in that of all tissues and systems. In addition, aberrant Notch signaling (both hyper- and hypoactive) has been implicated in a number of human developmental disorders (i.e. Alagille syndrome or cerebral autosomal dominant arteriopathy _CADASIL disease; see Box 2) and many cancers (see Box 3). Recently, it is also emerging its involvement in age-related diseases, by manifesting itself pleiotropic effects and acting through mechanisms not completely clear.

Here, we will report critical genes and signaling pathways involved in the ageing of stem and progenitor cells, focusing the interest on the role of Notch pathway (which seems to act by hub in an intricate signaling pathway network) and the related main mechanisms. In addition, we will discuss on the possibility to use Notch pathway, the related molecules and pathways constituting a network, both as innovative (predictive, diagnostic and prognostic) biomarkers and targets for personalised therapies of age-related diseases, which are the very challenges for the maintenance of heath in our populations. The concepts stressed, here, are fruit of considerations derived by expert opinion on the findings from author's studies on ageing, age-related diseases (particularly CVDs) and inflammation.

\section{Critical genes and signaling pathways in stem cell ageing and rejuvenation}

It is well recognized that the decline of tissue regenerative potential accompanies ageing. It may be due to age-related changes in tissue-specific stem cells. Adult stem cells, which divide throughout the life of an individual, experience both chronological and replicative ageing (Charville and Rando, 2011). This determines an enhance of the burden of mutations with age, as well as an increase in probability of cells, and particularly of stem and progenitor cells, to undergo apoptosis, malignant transformation, or senescence (Kuilman et al., 2010). The underlying cause of 
Box 2: Notch defects as causes of hereditary disorders and some diseases related to the spectrum of alterations regarding angiogenesis, vascular development and aortic valve anomalies.

In human, congenital cardiac malformation, Alagille syndrome, an autosomal dominant disorder characterized by developmental abnormalities, is caused by mutations in Notch ligand JAGGED1 gene. Another human disease, CADASIL (cerebral autosomal dominant arteriopathy with subcortical infarct and leukoencephalopathy) is related to a mutation in gene of the Notch-3 receptor and affects vascular smooth muscle cell (VSMC) development. Genetic mutation analysis of mice lacking of Notch receptors or their ligands revealed abnormalities during blood vessel formation, arteriovenous specification (You et al. 2005), and impaired proliferation and migration of endothelial cells (ECs) (Iso et al. 2003). Loss of Notch-1, Notch-4 (Krebs et al. 2004), Notch-3 (Domenga et al. 2004), Delta-like ligand (DII)-4 (Gale et al. 2004), or Jag-1 (Xue et al. 1999) lead to defects in angiogenesis and vascular development. Likewise, the knockout of Notch transcriptional targets Hey1 and Hey2 impaired vascular development, with vessels still forming de novo, but remaining small and failing to develop (Fischer et al., 2004). Notably, the Notch ligand DII-4 has been shown to be a pivotal regulator of tumor angiogenesis and vascular development, linking EC bioactivity to the tissue environment via Notch ligand receptor signaling (Noguera-Troise et al., 2006; Ridgway et al., 2006). In 2005, Garg and coworkers, demonstrated that mutations in the signaling and transcriptional regulator of Notch-1 cause a spectrum of developmental aortic valve anomalies and severe valve calcification in non-syndromic autosomal- dominant human pedigrees. Consistent with the valve calcification phenotype, Notch-1 transcripts were most abundant in the developing aortic valve of mice, and Notch-1 repressed the activity of Runx2, a central transcriptional regulator of osteoblast cell fate. The hairy-related family of transcriptional repressors (Hrt), which are activated by Notch-1 signaling, physically interacted with Runx2 and repressed Runx2 transcriptional activity independent of histone deacetylase activity. The authors hence suggested that Notch-1 mutations cause an early developmental defect in the aortic valve and a later de-repression of calcium deposition that causes progressive aortic valve disease. The same research group and others interestingly revealed that mutations in Notch-1 are also associated with congenital heart defects such as bicuspid aortic valve diseases (Niessen and Karsan, 2008).

\section{Box 3: Notch signaling in cancer.}

A significant association between the deregulation of the Notch pathway and different cancers has been observed (Ntziachristos et al., 2014). The mechanisms involved seem to be diverse. However, it sustains that they prevalently are the result both of processes of iperactivation or inactivation induced by mutations or other DNA/chromosome changes, but also of epigenetic alterations (Ntziachristos et al., 2014). For example, in $55-60 \%$ of the total T-cell acute lymphoblastic leukemia, mutations with activator biological effects, or deletions determining stop codons or frame shift effects have been significantly observed (Weng et al., 2004). In case of melanoma, it has been observed that Notch-1, in constitutive active form, mediates melanoma cell growth, by inducing betacatenin activation (Balint et al., 2005). In addition, the group of Ntziachristos underlined that an up-regulated Notch signaling has significant oncogenic effects in the onset of several neoplasia, including the related mechanisms to the inhibition of apoptosis process and induction of cellular proliferation (Ntziachristos et al., 2014). ageing, which determines all these changes, actually remains one of the central mysteries of biology. Despite this, recent studies in several different systems suggest, that not only may the rate of ageing be modified by environmental and genetic factors, but also that the ageing clock can be reversed, restoring characteristics of youthfulness to aged cells and tissues (Rando and Chang, 2012). An example has been provided in 2005 by the group of Rando on ageing of skeletal muscle stem cells (Conboy et al., 2005). Precisely, they examined the influence of systemic factors on aged progenitor cells from muscle tissues, by establishing parabiotic pairings (that is, a shared circulatory system) between young and old mice (heterochronic parabioses) and exposing old mice to factors present in young serum. Notably, heterochronic parabiosis restored the activation of Notch signaling as well as the proliferation and regenerative capacity of aged satellite cells. Thus, they concluded that the age-related decline of progenitor cell activity can be modulated by systemic factors that change with age. Another group, using the same type of investigations, i.e. parabiosis experiments, in 2014 demonstrated that the circulating protein growth differentiation factor 11 (GDF11) is a rejuvenating factor for skeletal muscle. As result, they suggested that GDF11 systemically regulates muscle ageing and may be therapeutically useful for reversing age-related skeletal muscle and stem cell dysfunction (Sinha et al., 2014).

Several signaling pathways have been identified to revert the process of skeletal muscle senescence. For example, the activation of Notch pathway has been shown to restore the myogenic differentiation capacity of satellite cells, bringing it to a level similar to that characterizing young cells from 20-years old humans (Carlson et al., 2009). On the other hand, with age, skeletal muscle stem cells have been shown to have markedly impaired capability of proliferation and differentiation into myoblasts (Conboy et al., 2003). Of note, cellular senescence in skeletal muscle stem cells may be due to diminished activation of Notch and its ligand Delta. Forced activation of Notch restored regenerative potential to old muscle (Conboy et al., 2003). In addition to the loss of Notch activation, it has been demonstrated the key role of transforming growth factor (TGF)- $\beta$. Old muscles have been shown to produce excessive levels of TGF- $\beta$, which interferes with their regenerative capacity by inducing TGF$\beta$ and pSmad3 in skeletal muscle stem cells (Carlson et al., 2008a, 2008b, 2009). Importantly, endogenous Notch and pSmad3 have been shown to antagonize each other in the control of satellite-cell proliferation, and attenuation of TGF-beta/pSmad3 in old, injured muscle restored regeneration to satellite cells (Carlson et al., 2009).

Recently, it has been also suggested that ageing can also be reversed in cardiovascular stem cells. Indeed, stem/progenitor cells in older humans are expected to have a decreased reparative capacity in response to tissue injury (as summarized in Madonna et al., 2016a, 2016b). Several molecules can be implicated in reverting the stem cell ageing, including Pim-1 kinase, Notch signaling and telomerase. In particular, Notch pathway plays also important roles in cardiac differentiation and regeneration. In mice, it has been shown that Notch is required for cardiac development and promotes the expansion of cardiac precursor cells (Yang et al., 2012; Nemir et al., 2014; Zhao et al., 2014a,b). Moreover, in zebrafish, it has been demonstrated that Notch signaling is required for cardiac regeneration (Zhao et al., 2014a,b). Pim-1 kinase has been identified as an anti-senescence factor in cardiac stem cells (CSCs) as well as in mesenchymal stem cells (MSCs). Pim-1 enhances proliferation (Fischer et al., 2009), metabolic activity (Mohsin et al., 2013) and differentiation (Choudhery et al., 2012; Zippo et al., 2004) of CSCs and mesenchymal stem cells (MSCs) in neovessels and new cardiac myocytes. Pim-1 also serves as a pro-survival mediator by preserving mitochondrial integrity (Borillo et al., 2010) and antagonizing intrinsic apoptotic cascades (Yan et al., 2003). Moreover, Pim-1 preserves telomere length and telomerase activity of CSCs (Mohsin et al., 2012). Finally, Mohsin and colleagues have shown that genetic 
modification of aged human cardiac progenitor cells (CPCs) with Pim-1 kinase results in remarkable rejuvenation of the CPCs associated with enhanced proliferation, increased telomere lengths, and decreased susceptibility to replicative senescence (Mohsin et al., 2013) (for extensive review on this topic, see Madonna et al., 2015, 2016a, 2016b).

Another pathway involved in stem cell ageing is the telomeretelomerase axis (for extensive review on this topic, see Balistreri et al., 2012, 2013, 2014). In 1998, it had been suggested that the life-span of human cells can be extended by telomerase activation (Bodnar et al., 1998). Since then great effort has been invested to translate this finding to cardiac cells (Wong et al., 2009; Brandt, 2010). Telomerase has been demonstrated to maintain telomere length, to contribute to cell survival and proliferation, and to prevent cellular senescence (Qu et al., 2011; Jan et al., 2012). In a subpopulation of adipose tissue-derived mesenchymal stromal cell MSCs (AT-MSCs), high levels of myocardin (MYOCD) and the catalytic subunit of telomerase (i.e., telomerase reverse transcriptase or TERT) with antisenescence properties have been detected (Madonna et al., 2008, 2013). MYOCD is a key regulator of cardiovascular myogenic development and nuclear co-transcription factor for myogenic genes, as well as genes involved in muscle regeneration and protection against apoptosis (Madonna et al., 2008, 2013). AT-MSCs have been shown to contain a population of adult multipotent mesenchymal stem cells with high cardiovascular regenerative potential. AT-MSCs that co- express TERT and MYOCD are characterized by high endogenous levels of octamerbinding transcription factor 4, MYOCD, myocyte-specific enhancer factor 2c, and homeobox protein Nkx2.5, as well as decreased cell death both in the form of spontaneous cell death and Fas-induced apoptosis. Therefore, TERT and MYOCD may act together to protect AT-MSCs from apoptosis and to enhance their cardiovascular myogenic development (Madonna et al., 2008, 2013).

Thus, a crucial role of Notch and other pathways emerges from these discoveries. However, their considerable relevance might be perturbed by evidencing that cellular senescence in the major number of these investigations, and precisely in cell culture experiments, can be induced not effectively by biological ageing, but likely by effects linked to extended cell cultures and passage number changes in muscle, cardiac or in any other type of cells. Consequently, cellular senescence can be likely caused by cell culture artefacts, as consequences of abnormally high levels of $\mathrm{O} 2$ and mismatch between the cells and their niches. Confirmations by other type of investigation are, therefore, needed.

\section{Molecular features of Notch signaling pathway}

In Caenorhabditis elegans, Notch pathway is functionally redundant, as it is encoded by the two genes, lin-2 and $g l p-1$, encoding for two different Notch proteins. The redundancy of Notch is due to the fact that GLP-1 is able to substitute for LIN-12 in cell fate decisions. Unlike Drosophila, which has only one Notch protein, vertebrates including mammals are characterized by redundancy too, as they have four genes encoding for four different interchangeable Notch proteins. Notch protein is constituted by a single-pass transmembrane protein of $300 \mathrm{kDa}$ molecular weight and 2500 aminoacids, composed of functional extracellular (NECD), transmembrane (TM), and intracellular (NICD) domains and characterized by two subunits called Epidermal Growth Factor (EGF) and Lin-Notch repeats (LNR) (Brown et al., 2000) (see details described in Box 4).

Notch signaling is initiated when Notch receptors EGF and LNR on the cell surface engage ligands presented in trans on opposing cells. Despite the expansive size of the Notch extracellular domain, it has been demonstrated that EGF domains 11 and 12

\section{Box 4: Structure of Notch pathway and canonical lig- ands.}

The Notch gene was discovered almost 90 years ago by Morgan and colleagues who observed that partial loss of function results in notches at the wing margin in flies (Drosophila melanogaster) (Borggrefe and Oswald, 2009). Decades later in loss of function experiments, Notch was found to cause a "neurogenic" phenotype, where cells destined to become epidermis switch fate and give rise to neural tissue, reviewed in (Borggrefe and Oswald, 2009). In the early eighties, the Notch gene was found to encode a $300 \mathrm{kDa}$ single-pass transmembrane receptor. The extracellular domain of the Notch receptor contains 36 epidermal growth factor (EGF)-like repeats essential for ligand binding. The intracellular domain is involved in cellular signaling and contains multiple conserved protein domains. Subsequently, Notch-like molecules have been identified from C. elegans (LIN-12) to humans, playing important, and apparently conserved, functional roles in development. In mammals, four Notch receptors (Notch 1-4) and five activating canonical ligands (JAGGED1, JAGGED2, DLL1, DLL3 and DLL4) have been described. Notch receptors are synthesized as single-chain precursors that, after glycosylation by protein O-fucosyl transferase (POFUT1) in the endoplasmic reticulum, are processed into noncovalently linked Notch extracellular (NECD) and intracellular (NICD) domains in the trans-Golgi (Caolo et al., 2011). Ligand binding induces a conformational change that allows the first proteolytic cut by "A Disintegrin And Metalloprotease," ADAM (the principal involved are 10 and 17). ADAM removes the extracellular portion of Notch and creates a membrane-tethered intermediate, which is a substrate for $\gamma$-secretase, a cleaving protease complex. $\gamma$ secretase, in turn, generates the active form of Notch (Notch intracellular fragment, NIC), which translocates to the nucleus, where it binds the transcriptional factor CSL (CBF1, Suppressor of Hairless, Lag-1), also known as recombinant signal binding protein 1 for JK (RBP-JK). Such NIC binding displaces repressor molecules and promotes the recruitment of coactivator molecules. This in turn activates the transcription of specific Notch target genes, such as Hes (hairy/enhancer of split), Hey (Hes-related proteins), Nrarp (Notch-regulated andrin repeat protein), cMyc, cyclin D1, and many other genes. Indeed, the spectrum of immediate notch targets is large and several genes may be activated in parallel, including those encoding repressor transcription factors like Snail1 (Sahlgren et al., 2008), p21 (Rangarajan et al., 2001) or c-Myc, with a context-dependent activator or repressor function (Weng et al., 2006).

are the critical determinants for interactions with ligands. Additional studies have implicated regions outside of Notch EGF11-12 in ligand binding. For example, Notch EGF domain 8 plays a role in selective recognition of ligands such as Serrate/Jagged, while EGF domains 6-15 are required for maximal signaling upon ligand stimulation. In mammalian signal-sending cells, members of the Delta-like (DLL1, DLL3, DLL4) and the Jagged (JAG1, JAG2) families serve as ligands for Notch signaling receptors. Upon ligand binding to EGF, the NECD is cleaved away from the TM-NICD domain by TACE (TNF- $\alpha$ disintegrin and metalloprotease (ADAM) metalloprotease converting enzyme). This proteolysis of the Notch receptor is in the ER and Golgi. This cleavage occurs through proteolysis at the level of $\operatorname{Tyr}_{1605}-\mathrm{Met}_{1606}$ residues by the metalloproteinase ADAMT. NECD is then endosome-transported to the plasma membrane to enable ligand binding in a manner regulated by Deltex and inhibited by NUMB. The processed receptor NECD remains bound to the ligand and this complex is then endosome-transported to the plasma membrane to enable ligand binding in a manner regulated by Deltex and inhibited by NUMB. NECD also undergoes endocytosis/recycling within the signal-sending cell in a manner dependent on ubiquitination by Mib. In the signal-receiving cell, 
Table 1

Target genes for Notch in mammals.

\begin{tabular}{|c|c|}
\hline Target genes & Cell function \\
\hline CDKN1A, CFLAR (CASH), IL2RA, NFKB1 & Apoptosis \\
\hline CCND1, CDKN1A, IL2RA & Regulators of cell cycle \\
\hline CDKN1A, ERBB2, FOSL1, IL2RA & Regulators of cell proliferation \\
\hline DTX1, PPARG & Regulators of cell differentiation \\
\hline HES1, HEY1 & Neurogenesis \\
\hline $\begin{array}{l}\text { DTX1, FOS, FOSL1, HES1, HEY1, NFKB1, } \\
\text { NFKB2, NR4A2, PPARG, STAT6 }\end{array}$ & Regulators of Transcription \\
\hline $\begin{array}{l}\text { CD44, CHUK, IFNG, IL17B, KRT1, LOR, } \\
\text { MAP2K7, PDPK1, PTCRA }\end{array}$ & Unspecified functions \\
\hline GLI1, GSK3B, SHH, SMO, SUFU & Interaction with Sonic Hedgehog (Shh) Pathway \\
\hline $\begin{array}{l}\text { AES, AXIN1, CTNNB1, FZD1, FZD2, FZD3, } \\
\text { FZD4, FZD6, FZD7, GSK3B, LRP5, TLE1, } \\
\text { WISP1, WNT11. }\end{array}$ & Interaction with Wnt receptor signaling pathway \\
\hline
\end{tabular}

CANONICAL LIGAND

NON CANONICAL LIGANDS

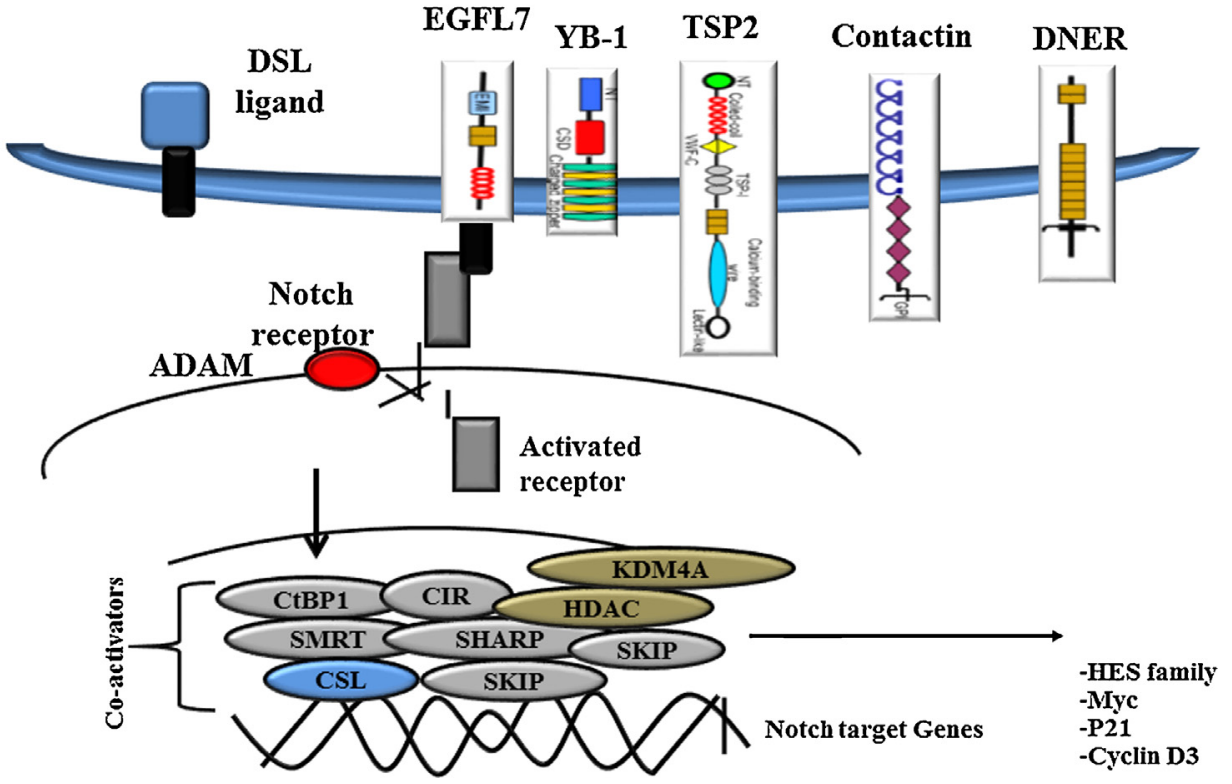

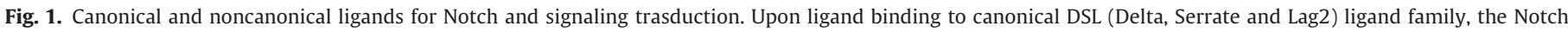

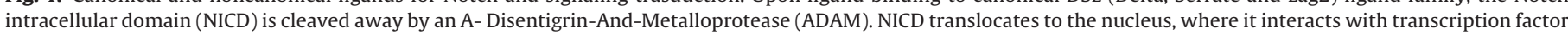

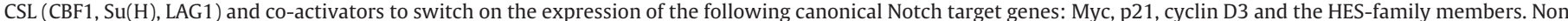

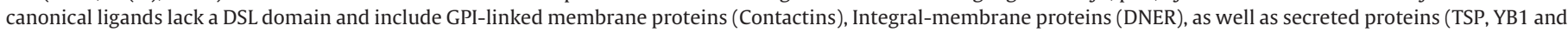

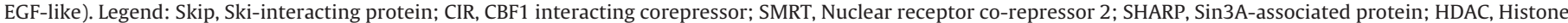
deacetylase; KDM4A, lysine-specific demethylase 4A; EGF, endothelial growth factor; DNER, Delta/Notch-like EGF-related receptor; TSP, thrombospondin.

$\gamma$-secretase (also involved in AD) releases the NICD from the transmembrane domain TM. NICD can enter the nucleus and start to orchestrate changes in DNA-bound protein assemblies. In particular, NICD in the nucleus forms a complex with the nuclear protein CBF1/Su(H)/LAG-1 (CSL) transcription factor complex, resulting in subsequent activation of the canonical Notch target genes: Мус, p21, and the HES-family members. This bond prevents CSL to interact with several transcriptional co-repressor complexes, with the result that CSL becomes transcriptionally active. NICD/CSL complex interacts with co-activators of the Mastermind-like (MAML), which is essential for the recruitment of the Histone acetyltransferases (HAT) protein $\mathrm{p} 300$. The HAT/Notch/CSL complex would acetylate histones, increasing the likelihood for the formation of an open chromatin and an active transcription complex. HAT/Notch/CSL complex is followed by up-regulation of downstream target genes belonging to Hairy/Enhancer of Split (HES) family of basic helix-loop-helix transcriptional regulators (Wu et al., 2000). Table 1 summarizes target genes identified to date.

\section{4. "The paradigm on versatility of cell responses mediated" by Notch pathway}

As above mentioned, Notch signaling receptor pathway plays an important role in normal embryonic development of all metazoan species, by controlling a broad range of developmental processes, including cell fate determination, proliferation, migration and terminal differentiation. In addition, it has key functions in maintaining stem cells in adult, as well as in regulating homeostasis of all tissues (Fiúza and Arias, 2007). Surprisingly, current evidence also sustains a key role of Notch pathway in the patho-physiology of a large range of age-related diseases, from CVDs to AD (Carlson et al., 2008a, 2008b; Talora et al., 2008; Alberi et al., 2013; Aquila 
Box 5: Non-canonical Notch ligands.

One possible explanation of the pleiotropic nature of Notch signaling is due to the detection of non canonical ligands. Unlike canonical ligands, which share many features, non canonical ligands are structurally diverse and include integral membrane, GIP-linked, and even secreted proteins. One of the earliest described non-canonical ligands for Notch is Delta-like 1 (Dlk-1), also known as Pref-1, or FA-1, whose predominant role is inhibiting adipogenesis. Other than the lack of a DSL domain, DIk-1 is otherwise quite similar in structure to other Delta-like proteins, as it is an integral membrane protein containing tandem EGF repeats in its extracellular domain. Moreover, like Delta, Dlk-1 can be cleaved by ADAMs and is negatively regulated at the transcriptional level by Notch signaling. The preponderance of evidence supports only cis-interactions between Dlk-1 and Notch, and in fact, Dlk-1 overexpression phenotypes are consistent with Dlk-1 functioning only in cis-inhibition and not trans-activation of Notch signaling. Dlk-1 cis-inhibition may depend on the amount of ADAM proteolysis, since an ADAM-resistant, membrane-bound form of Dlk-1 is more potent than wild-type or soluble forms at blocking Notch signaling. This suggests that Dlk-1-mediated Notch antagonism may require low cellular ADAM activity, that favors membrane-bound Dlk-1. High levels of Dlk-1 are also associated with loss of Notch target gene expression such as Hes-1 and E(spl)m $\beta$ in mammals and flies, respectively. The molecular basis of this antagonism is unclear, but it is possible that Dlk-1 binding to Notch EGF 10-11 or EGF 12-13 may compete with activating trans-DSL ligand that requires Notch EGF 11-12 to block binding and signaling. However, direct binding of full-length DIk-1 and Notch, either endogenously or ectopically expressed, has not been reported. Moreover, there is conflicting data on whether Dlk-1-induced loss of Hes-1 expression directly involves Notch, since Hes-1 is regulated by more than one signaling pathway. Another Delta-like protein is Delta/Notch-like EGF-related receptor (DNER), which is an integral membrane protein containing extracellular tandem EGF repeats but lacking a DSL domain. Despite the absence of a DSL domain, DNER binds Notch when presented in trans and can activate a CSL reporter in cells co-cultured with DNER-expressing cells. Both in vitro and in vivo studies support DNER's function as a trans-ligand to effect glial morphological changes through activation of Notch. DNER does not affect the number of glial cells present in vivo, suggesting that its effect is limited to later stages of differentiation and not early cell fate decisions. DNER is expressed in Purkinje cells, where it is available to activate Notch in the adjacent Bergmann glia, and indeed DNER mutant mice show morphological defects in Bergmann glia. Soluble DNER (DNER-Fc) can also affect Bergmann glia morphology in vitro in a $\gamma$-secretase-dependent, but CSL-independent manner, suggesting that Notch proteolysis plays a role in this process, but not to generate a transcriptional co-activator for CSL proteins. Instead of CSL, the E3 ubiquitin ligase Deltex has been implicated as an alternative downstream effector of Notch through in vitro studies in which a dominant-negative form of Deltex blocked the DNERinduced morphological changes. Deltex can bind directly to the Notch intracellular domain, and mediate a trimeric complex between itself, full-length Notch, and $\beta$-arrestin, making it possible that Notch could activate signaling through $\beta$-arrestin that would require Deltex but not CSL. One caveat of DNER function as a non-canonical ligand is that its effects have not been formally shown to require Notch receptor expression in Bergmann glia. Recently, a putative DSL ligand-like protein called Jagged and Delta protein (Jedi) was reported based on sequence data. However, upon closer examination, the putative DSL and EGF repeats of Jedi do not contain the conserved cysteine spacing common to either the signature motif of canonical ligands or EGF repeats that are also present in DNER and Dlk-1. Instead, the Jedi extracellular domain contains an N-terminal emilin domain followed by multiple tandem repeats of an 8-cysteine variation of the EGF domain interspersed with two single 6-cysteine EGF repeats. In fact, Jedi has neither trans-activating nor cis-inhibitory activity, and has not been reported to interact with any of the Notch receptors. Although soluble Jedi added to Notchexpressing cells weakly inhibits a Notch reporter, there is currently no strong evidence linking Jedi to Notch signaling. Structurally distinct from the integral membrane non-canonical ligands are F3/contactin1 and NB3/contactin6, that encode GPI-linked neural cell adhesion molecules. Both contactins have been reported to activate Notch signaling to induce oligodendrocyte (OL) differentiation. Binding and fractionation studies indicated that either contactin could interact with Notch in trans, although cis interactions cannot be ruled out since both endogenous F3 and NB3 co-immunoprecipitate with Notch (and vice versa). Both contactins interact with Notch EGF repeats distal to the DSL binding site, while only F3 can interact with Notch EGF repeats 1-13, which contain the DSL ligandbinding site at EGF 11-12. While this interaction makes it possible that F3 competes for the DSL ligand-binding site, further studies will be required to determine whether the F3 and DSL binding sites actually overlap. Similar to DSL ligand treatment, adding soluble forms of either contactin to OL cells produces NICD in a $\gamma$-secretase-dependent fashion, which can translocate to the nucleus for signaling. However, downstream of NICD generation, contactin-based signaling does not appear to involve CSL. F3-Notch signaling does not activate Hes-1 transcription, and there are no reports on the ability of NB3 to activate canonical CSL-induced Notch signaling. Instead of CSL, the contactins both induce Notch signaling that involves Deltex to induce glial maturation. An interesting dichotomy is raised in these in vitro assays in which the same cells (and presumably the same Notch receptors) differentiate in response to contactins and remain progenitors in response to DSL ligand or NICD expression. It is thought that temporal regulation of DSL ligand and contactin expression may regulate in vivo which effect takes precedent as DSL ligands are expressed early in embryonic development while contactins are highly expressed only after birth. Therefore, like DNER, the contactins appear to utilize Notch to effect changes late in differentiation as opposed to DSL ligands that can impact early cell fate decisions (as reviewed in D'Souza et al., 2008, 2010).

et al., 2013). This denotes its capacity to mediate a multitude of cellular responses and pleiotropic effects, which certainly reflect not only the complexity and fundamental features of this pathway. Notch pathway shows, indeed, the following features: (i) a relatively simple structure constituted by a limited number of obligatory components, organized to form a core pathway (Mumm and Kopan, 2000; Borggrefe and Oswald, 2009); (ii) the major number of its actions are evocated and only mediated by the five canonical ligands, which unusually are also transmembrane proteins (D'Souza et al., 2008); (iii) the intracellular transduction of the Notch signal is remarkably simple, with no secondary messengers; and (iv) the identification of non-canonical ligands has recently expanded the repertoire of ligands reported to activate signaling (D'Souza et al., 2010) (see Boxes Box 4 and Box 5; and Fig. 1). Thus, such function 's versatility probably comes from other factors. Among the potential candidates, extrin- sic and intrinsic mechanisms seem the most plausible, modulating the Notch signaling and likely contributing to the versatile output. Accordingly, in 2006, Bray underlined for the first time that "Notch is a simple pathway, but it becomes complex in its actions" (Bray, 2006). Co-temporally, Bray and other researchers suggested that several mechanisms can modulate the activity of the Notch pathway in a positive or negative manner (as reviewed in Bray, 2006; Poellinger and Lendahl, 2008; Fior and Henrique, 2009; Borggrefe and Oswald, 2009; Wang et al., 2015). However, how they are integrated to regulate the temporal, spatial and directional aspects of cell fate decision is still not completely understood.

In following paragraphs, some relevant aspects emerged by these studies will be pointed, before discussing about potential Notch mechanisms, which are most probably associated with agerelated diseases. Advances in understanding this cascade are highly 
relevant, not only for basic science, but also for clinicians, since they offer a concrete potential in developing therapeutic strategies to counteract alterations in Notch signaling, and consequently to retard or delay age-related diseases.

\subsection{Notch pathway as rheostat}

The most acceptable opinion about Notch signaling is that it functions as a rheostat, which can be adjusted to produce different intensities in the signaling output. In particular, the intensity of signaling output mediated by Notch pathway can derive by the diverse density of expression of tissue-specific ligands, or by varying levels of Notch pathway. As a consequence, Notch can influence the fate and decision of a variety of cell types, and it can contribute to tissue patterning and morphogenesis by affecting cell differentiation, proliferation, survival and apoptosis. On the other hand, this concept is confirmed by the findings of studies on lymphoid differentiation performed by group of Bernstein. Thus, the density of Notch ligands associated or not with the different Notch expression levels in different tissues, organs and systems may be an important determinant in regulating cell-fate outcomes (Delaney et al., 2005). Co-temporally, this evidence underlines another critical point, that perturbations in intensity of levels of activation Notch signaling may result in the evocation of disease (as above mentioned). For example, defects in Notch ligands are associated with Alagille syndrome, spondylocostal dysostosis, cerebral autosomal dominant arteriopathy, while an aberrant ligand Notch expression is detected in several cancers (see Boxes 2 and 3). Likewise, mutations on Notch genes are related to the spectrum of alterations regarding angiogenesis, vascular development and aortic valve anomalies (i.e. bicuspid aortic valve) (Garg et al., 2005; Niessen and Karsan, 2008) (see Box 2).

\subsection{The regulation of notch signaling rheostat is \\ "cell-context-dependent" and mediated by several mechanisms}

Researchers involved in the Notch studies often get out of complicated discussions on the paradigm of versatility of cell responses mediated by using a common expression or saying: "but of course, this aspect of Notch signaling is cell-context-dependent". It is important to reinforce that this is not always just a handwaving explanation, and that Notch, indeed, elicits very different responses, depending on the cellular context. It is, therefore, important to understand how Notch signaling is tuned-up, both in the normal development and diseased conditions. It has been demonstrated that Notch is regulated by multiple mechanisms. At first, Notch controls a much larger number of genes in a direct manner than the few Hes/Hey genes initially proposed (Monastirioti et al., 2010) (see Table 1.). For example, a large set of transcription factors are regulated in a cell-context specific way along with the canonical Hes/Hey genes, but also in parallel with the b-HLH protein of the Id family, which can block the activity of other b-HLH proteins (as summarized in Wang et al., 2015). Since Hes/Hey proteins can block lineage specific transcription factors, the Id proteins, in turn, can inhibit Hes/Hey proteins. This is a good example of an incoherent logic network, as proposed by the Bray group (see above). Here, Notch signaling results in an ambiguity of the outcome, since different targets block each other, leaving the network in a poised state. Thus, the intrinsic or extrinsic cell-context, also influenced by other signaling pathways, could be the decisive factor to swing to one or the other side of a decision. On the other hand, the group of Borggrefe has recently described in an exhaustive manner the crosstalk of Notch pathway with other evolutionally conserved signaling pathways, like the Wnt, Hedgehog, hypoxia and TGFß/BMP pathways, which can affect Notch signaling output (Borggrefe et al., 2016). This regulation can happen by controlling ligand, receptor or transcription factor expression, regulating the protein stability of intracellular key components, usage of the same cofactors or co-regulation of the same key target genes (as summarized in Borggrefe et al., 2016).

In addition, Melino s' group has demonstrated that, acting as a key molecule in embryonic development, p63, the most ancient member of the p53 family of transcription factors (Yang et al., 1998), can regulate components of Notch pathway through the direct transcriptional induction of Jag1 and Jag2 during epidermal differentiation or thymic development (Candi et al., 2007; Terrinoni et al., 2013). Co-temporally, the same group also evidenced that Cpromoter binding factor-1 (CBF-1)-dependent gene transcription driven by the Notch-1 intracellular domain (N1(ICD)), is potently antagonized by exogenously expressed trans-activating (TA) p73 splice variants in SH-SY5Y neuroblastomas and in primary neurones. Time course analyses have also shown that the inhibitory effects of TAp73 are direct and not mediated via the product of a downstream target gene. Thus, endogenous TAp73 stabilized by either c-Abl or cisplatin treatment, also potently antagonized N1(ICD)/CBF-1-dependent gene transcription. Furthermore, western blotting revealed that exogenous TAp73 suppressed endogenous hairy and enhancer of split-1 (HES-1) protein levels and antagonized the increase in HES-1 protein induced by exogenous N1(ICD) expression. Evidence of a direct physical interaction between N1(ICD) and TAp73alpha has been demonstrated by performing co-immune-precipitation. Using Notch deletion constructs, Melino and coworkers demonstrated that TAp73alpha binds the N1(ICD) in a region C-terminal of aa 2094. Interestingly, DeltaNp73alpha and TAp73alpha(R292H) also co-purified with N1(ICD), but neither inhibited N1(ICD)/CBF-1-dependent transcription. This suggests that an intact trans-activation (TA) domain and the ability to bind DNA are necessary for TAp73 to antagonize Notch signalling. Finally, TAp73alpha reversed the N1(ICD)-mediated repression of retinoic acid-induced differentiation of SH-SY5Y neuroblastomas, providing functional evidence for an inhibitory effect of TAp73alpha on Notch signaling.

Based on these relevant observations, which denote as Notch pathway mediates several functions by cross-acting with other conserved signaling pathways, we will illustrate, in the next paragraphs, the emerging evidence accumulated during the last years about the crosstalk of Notch pathway with the crucial innate/inflammatory pathways, recognized to have a role of hub in the complex patho-physiology of age-related diseases. For facilitating its comprehension, it firstly will report a brief description about ageing as the greatest risk factor for the development of age-related diseases.

\section{Ageing as the key driver of both age-related inflammation and the development of age-related diseases: focus on a large complex network of pathways. Notch pathway included?}

Ageing is a complex process, induced by an intricate interaction of genetic, epigenetic, stochastic and environmental factors. They determine the lost of molecular fidelity followed by an improved entropy (Hayflick, 2007). As result, loss of complexity and random accumulation of damages (i.e. particularly damages to nuclear and mitochondrial DNA) at cellular, tissue, organ levels and/or of whole body arise. Thus, it establishes a condition, which modifies both architecture and functioning of physiological processes and regulatory (immune and endocrine) systems (Salminen and Kaarniranta, 2010). This determines a deterioration of the homeostasis. Accordingly, it becomes more easily vulnerable to internal and external stressors, frailty, disability and disease. In particular, host defence evocates, as response of damage, the vigorous 
activation and participation of a complex inflammatory network (Vasto et al., 2007), which determine catastrophic innate/inflammatory reactions, responsible of massive release of so-called senescence associated secretory phenotype (SASP), represented by a myriad of factors, such as the pro-inflammatory mediators (revised in Balistreri et al., 2013). In turn, SASP, which occurs in several cells (i.e. fibroblasts, epithelial cells, ECs, astrocytes, preadipocytes, and leukocytes as well as in post-mitotic cells) creates and feeds a low chronic grade of inflammation, called "inflamm-aging", by improving both entropic ageing process and onset risk for age-related degenerative diseases, as well postulated for the first time by Franceschi in 2000 (Franceschi et al., 2000). Thus, the persistence of stimuli, including immune insults, external and internal danger signals associated with senescence and ageing process (i.e. oxidative and genotoxic stress and tissue injuries), and the consequent "chronic sterile inflammation" without pathogens predispose individuals to various age-related diseases (Chung et al., 2009). For example, the pro-inflammatory SASP of senescent EC cells has been proposed to contribute to CVDs, by initiating and fuelling the development of atherosclerotic lesions, but also the vascular remodeling and medial degeneration associated with other pathological conditions, i.e. aneurysms (as summarized in Ovadya and Krizhanovsky, 2014; Balistreri, 2015). In addition, the expression of a SASP by astrocytes, documented both in cells that were made senescent in culture as well as cells that were isolated from aged brain tissue, has been suggested to initiate or contribute to neuroinflammation, responsible of many neurodegenerative diseases, such as $\mathrm{AD}$, causing or exacerbating age-related decline in both cognitive and motor function (as summarized in Campisi et al., 2011; Ovadya and Krizhanovsky, 2014).

As above mentioned, both SASP and the chronic sterile inflammation are the result of the activation and participation of a complex inflammatory network (as summarized in Vasto et al., 2007; Balistreri et al., 2013). Namely, several recognition pathways, i.e. Toll-like receptors (TLRs) and inflammasome, as well as different upstream kinase cascades, via canonical or non-canonical pathways, are involved, and all linked to the nuclear factor (NF)- $\kappa$ B pathway (as summarized in Balistreri et al., 2013). Among these, the TLR-4, one of the best understood TLR members, is able to recognize pathogen-associated molecular patterns (PAMPs) (e.g. the lipopolysaccharide, LPS), but also endogenous danger molecules, the DAMPs, such as heat-shock proteins (HSPs), hyaluronic acid, $\beta$-defensin-2, oxidized-LDL (ox-LDL), fibronectin, and amyloid peptide (Balistreri et al., 2009). In addition, it has been demonstrated a key role of TLR-4 in the pathogenesis of several age-related diseases (as summarized in Balistreri et al., 2009). Accordingly, intense investigations from our research group on inflammation and agerelated diseases (i.e. CVDs, $\mathrm{AD}$, diabetes and prostate cancer) led to obtain relevant data gathered for over 10 years, which suggest the key involvement of an intricate network of innate/inflammatory pathways in their complex patho-physiology (as summarized in Vasto et al., 2007; Balistreri et al., 2009). In this network, our data suggest the role of TLR- 4 as hub (as summarized in Balistreri et al., 2009). Such findings have been recently confirmed by our group in another setting of age-related disease, such as sporadic thoracic aortic aneurysm (Ruvolo et al., 2014; Balistreri, 2015).

New recent insights and advances in the research on Notch pathway are suggesting its significant role even in the onset of several age-related diseases, from CVDs to AD (Carlson et al., 2008a, 2008b; Talora et al., 2008; Alberi et al., 2013; Aquila et al., 2013). However, the mechanisms involved in such role of Notch pathway remain poorly understood, even if confirmations and suggestions, from both animal and human studies, on its key role continue to increase.

As regards, our speculation is that their identification might derive by considering a fundamental event, which characterizes both the alteration of homeostasis of the tissues, organs, systems of the human body, as well as the changes associated with the inevitable ageing process and the onset of age-related diseases. Such event is represented by the macrophage phenotypic switch, from M2 to M1, and vice versa, discovered by Mantovani s' group (Sica and Mantovani, 2012). As well documented, it may be followed or not by the participation of other inflammatory cell types, such as T and B lymphocytes, which may have roles in the pathogenesis of inflammation and onset of age-related diseases, such as CVDs (as summarized in Vu et al., 2016). Accordingly, monocytes released from bone marrow into the circulation migrate into tissues of the body, where they differentiate into mature macrophages, including liver kupffer cells, brain microglia, lung alveolar macrophages, peritoneal macrophages, adipose tissue macrophages and bone osteoclasts, and EC cells (Perdiguero and Geissmann, 2015). Here, they have long been held to be served as potent immune effector cells with well established roles in both the tissue homeostasis and lesions, e.g. they promote inflammation, tissue remodeling(e.g. vascular remodeling), angiogenesis, tumour immunity and consequent progression of tissue injury observed in various age-related diseases, i.e. CVDs. Several lines of evidence have now revealed that the heterogeneity and plasticity of these conditions are hallmarks of macrophages (Gordon et al., 2014; London et al., 2013). In response to PAMPs and self-local environment-derived stimuli (DAMPs) in a generally TLRs (and particularly TLR- 4 mediated) and NF- $\kappa B-$ dependent fashion, macrophages can assume a spectrum of states ranging from classically activated M1 inflammatory macrophages, to various alternatively activated M2 macrophages, involved in immune regulation and tissue repair (Sica and Mantovani, 2012). M1 macrophages are characterized by production of inflammatory mediators, such as IL-12 and inducible nitric oxide synthase (iNOS), in response to PAMPs or DAMPs-mediated activation of TLRs and cytokines, such as interferon- $\gamma$ (IFN- $\gamma$ ). In contrast, M2 macrophages (induced by IL4/IL13 (M2a), immune complex (M2b), and the anti-inflammatory cytokines IL-10 or transforming growth factor- $\beta$ (M2c)), express less inflammatory mediators and play a key role in wound healing, host defence against helminths, and resolution of inflammation. Thus, the specifically microenvironmental factors shape macrophage properties and activation state, regulating the phenotypic and functional dynamic switch, which represents different extremes of a continuum ranging from M1 to M2 (Sica and Mantovani, 2012).

Noticeably, mounting evidence from a number of different studies in vivo and in vitro has generally indicated that identifying the activated states of macrophages and targeting the macrophage polarization from M1 to M2 or vice versa, might be served as novel diagnostic or therapeutic strategies for multiple age-related diseases. This interest is leading to identify the cellular signaling pathways involved, such as the Notch pathway. Here, we will describe the role of Notch pathway in monocyte/macrophage cells, their activation through the cooperation or not with TLR pathway, functions and polarization.

\subsection{Notch in monocyte/macrophage cells, activation and functions: its cooperation with TLR and NF- $\kappa B$ pathways}

It is well documented the role of Notch pathway and their ligands in regulating multiple steps of $\mathrm{T}$ and $\mathrm{B}$ cell development in both central and peripheral lymphoid organs (as summarised in Cruickshank and Ulgiati, 2010; and in Amsen et al., 2015). Less is known about the role of Notch pathway in regulating myeloid development and function, especially in the context of acute and chronic inflammation. However, recent progresses have firstly demonstrated that Notch pathway is involved in this process, even if there are discrepancies in mechanisms involved (Bigas and Espinosa, 2012). In particular, early studies using myeloid cell lines showed that forced Notch- 1 or Notch- 2 activation could 
inhibit myeloid differentiation in a cytokine-dependent manner (Milner et al., 1996; Bigas et al., 1998), likely through expression of Gata2 (Kumano et al., 2001). A similar result was recently reported by using isolated KSL population co-cultured on an OP9 stromal cell line expressing Delta1 (de Pooter et al., 2006). In apparent contradiction with in vitro data, the analysis of different conditionally-targeted Notch alleles showed that Notch activity is only required for $\mathrm{T}$ and $\mathrm{B}$ lymphoid differentiation in vivo (Radtke et al., 1999). Nevertheless, it has recently been reported that mice defective in the FX protein, that converts the GDP-mannose to GDP-fucose, which is required for Fringe-dependent glycosylation of Notch signaling, develop a myeloproliferative disease. This phenotype has been attributed to a defect in the Notch pathway since fucosylation-deficient myeloid progenitors were not able to respond to Notch-ligands and failed to differentiate compared to the wild type cells (Zhou et al., 2008). Together, these results suggest that Notch is dispensable for myeloid cell generation but its activity is required for the maintenance of myeloid homeostasis in vivo.

Besides its role in myeloid differentiation, an increasing evidence is supporting a role of Notch pathway in regulating activation and function of terminally differentiated myeloid cells. Macrophages and dendritic cells (DCs) constitutively express Notch ligands and receptors, as well as a variety of pattern recognition receptors (PRRs) including TLRs, with a modulation evocated through several mechanisms. One of these mechanisms is mediated by TLRs, which modulate Notch signaling, by inducing Notch receptor and ligand expression. There is large evidence that activation of macrophages and DCs with TLR ligands leads to induction of Notch receptors and ligands, including Jagged 1, Dll1, Dll4 (Amsen et al., 2004; Fung et al., 2007; Foldi et al., 2010; Monsalve et al., 2006; Monsalve et al., 2009; Palaga et al., 2008; Zhang et al., 2012). For example, it has been demonstrated that Dll4 (even if considered as an endothelial cell-specific Notch ligand) expression increased in macrophages exposed to pro-inflammatory stimuli such as LPS, IL-1 $\beta$, or minimally modified low-density lipoprotein (LDL) in TLR-4-NF- $\kappa B$ manner. In addition, it has been also demonstrated that Dll4 expression is crucial for triggering Notch proteolysis and activation, leading to the induction of proinflammatory molecules and pathway such as the typical M1 gene iNOS, mitogen -activated protein kinase, Akt and NF- $\kappa$ B. Furthermore, Dll4 ligation to macrophages promoted the expression of Dll4 itself. These findings led to hypothesize that Dll4 - Notch signaling mediates inflammatory responses by accelerating a positive feedback loop of macrophage activation, leading to onset to atherosclerotic vascular diseases, metabolic disorders or other inflammatory diseases (Shang et al., 2016).

Thus, a cooperation between TLR and Notch is required for an optimal activation of macrophages. To confirmation of such suggestion, an intense work from a number of laboratories suggested that the activation of Notch target genes is a common feature of TLR responses and can occur via two non mutually exclusive mechanisms, direct activation by acute TLR signaling and indirect activation secondary to Notch receptor and ligand induction. Accordingly, Hu s' group observed that activation of Notch target genes, such as Hes1 and Hey1, can be directly induced by TLR stimulation (Hu et al., 2008). However, the signals and molecules involved remain to be identified.

Another mechanism regulating Notch signaling in myeloid cells and other cell types seems to be linked to inflammatory cytokines, such as TNF- $\alpha$ and IL-1 $1 \beta$. For example, under uncontrolled inflammation, TNF- $\alpha$ induces Notch-1 and Notch4 expression on synovial fibroblasts (Ando et al., 2003). Another example of Notch expression mediated by TNF- $\alpha$ is observed in a mouse pancreatic cancer model, as demonstrated Maniati and collegues in 2011(Maniati et al., 2011). It has been reported that
IL-1 $\beta$ induces Notch targets expression in chondrocytes, by inducing Notch-1 activation with a similar action of TNF- $\alpha$ (Ottaviani et al., 2010). In addition of pro-inflammatory cytokines, TGF- $\beta$ has also been shown to directly induce Hes 1 expression. IFN- $\gamma$ functions as a negative regulator of Notch activation. In human macrophages, it has been demonstrated that Hes1- and Hey1 expression was abrogated by IFN- $\gamma$, which blocked TLR-induced activation of canonical Notch target genes by inhibiting Notch-2 signaling and downstream transcription. These findings identify new immune functions for RBP-J, Hes, and Hey proteins and provide insights into mechanisms by which Notch, TLR, and IFN- $\gamma$ signals are integrated to modulate specific effector functions in macrophages (Hu et al., 2008).

In summary, Notch target gene expression can be activated in myeloid cells by a wide array of inflammatory stimuli, including TLR ligands and cytokines. In addition, the logic pathway involved in the activation of Notch target genes is NF- $\kappa$ B signaling, as demonstrated in many systems as cancer (Espinosa et al., 2010). Moreover, another pathway is the mitogen-activated protein kinases (MAPKs) (Hu et al., 2008). In addition, three mechanisms seem to be involved: (i) transcription factor cooperation orchestrated by NICD that can directly interact with NF- $\mathrm{KB}$ subunits and promotes transcription (Osipo et al., 2008); (ii) release of inhibitory molecules: an example is given from resting cells inhibitor of NF- $\kappa B$ (IкB), which typically sequesters NF- $\mathrm{B}$ in cytoplasm. It has been found to be present at the promoter regions of Hes1. Interestingly, TNF-induced Hes1 expression is thought to be associated with dismissal of I $\mathrm{I} B \alpha$ from the Hes 1 promoter (Aguilera et al., 2004); and (iii) chromatin modification: TNF and TLR ligand-induced Hes 1 gene transcription has been associated with upregulation of positive histone marks, such as serine 10 phosphorylation and K14 acetylation of histone H3 at the Hes1 promoter (Aguilera et al., 2004; Hu et al., 2008; Maniati et al., 2011). Both IKKs and MAPKs have been implicated in mediating inflammatory signaling-induced chromatin modifications at the Notch target gene loci (Hu et al., 2008; Zeng et al., 2005; Aguilera et al., 2004). Taken together, NF-кB and MAPK signaling pathways appear to play a critical role in mediating Notch target gene activation by inflammatory stimuli.

\subsection{Notch in their polarization and potential mechanisms associated}

In the induction of inflammatory conditions, macrophages heterogeneity and plasticity are typical features, which lead to a gene expression for a determined phenotype and a consequent change of effector functions. Despite accumulating in vitro evidence and its large impact, in vivo mechanisms remain incompletely understood. Recent progresses have been achieved. Indeed, the Fukuda group has explored the role of Dll4-mediated Notch signaling in macrophage activation, since this Notch ligand is expressed on EC cells and endothelial dysfunction is correlated to its expression. To this aim, they assessed monocyte/macrophage polarization using antibody Dll4 treatment and stromal vascular fraction obtained from epididymal fat. The results obtained demonstrated that Dll4-mediated Notch signaling induces a macrophage polarization toward a proinflammatory phenotype (Fukuda et al., 2012). Similarly, the group of Pabois demonstrated the same role of Dll4mediated Notch signaling using as model the antibody-mediated rejection (AMR) of the cardiac allograft (Pabois et al., 2016). They investigated the mechanisms underlying vascular and inflammatory cell network involved in AMR at endothelial and macrophage levels, using endomyocardial transplant biopsies and EC/monocyte cocultures. First, they observed that AMR associates with changes in Notch signaling at endothelium/monocyte interface including loss of endothelial Notch 4 and the acquisition of the Notch ligand Dll4 in both cell types. They also showed that endothelial 


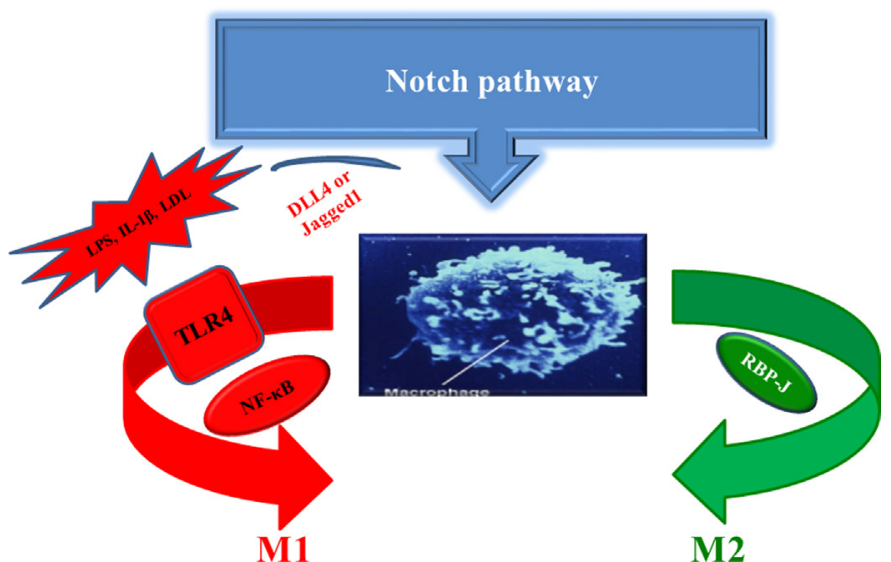

Fig. 2. Notch pathway in the macrophage phenotype polarization. Macrophage polarization is a process that is driven by stimuli of the surrounding microenvironment, including cytokines, lipoproteins, growth factors and bacterial lipopolysaccharide (LPS). LPS in particular activates M1 macrophage polarization by inducing toll like receptor (TLR)-4, which, in turn, can activate the nuclear factor $\kappa \mathrm{B}(\mathrm{NF}-\kappa \mathrm{B})$ pathway. This leads to the production of nitric-oxide (NO) through inducible NO synthase (iNOS). Activation of Notch results in M2 macrophage polarization, through the binding of the Notch intracellular domain (NCID) to the DNA binding protein immunoglobin kappa J (RBP-J), which controls the expression of IL-12 and iNOS. The M2 macrophage polarization is involved in the resolution of inflammation, angiogenesis and tissue remodeling.

Dll4 induces macrophage polarization into a pro-inflammatory fate $\left(C D 40^{\text {high }} \mathrm{CD} 64^{\text {high }} \mathrm{CD} 200 \mathrm{R}^{\text {low }}\right.$ HLA-DR $\left.{ }^{\text {low }} \mathrm{CD} 11 \mathrm{~b}^{\text {low }}\right)$, eliciting the production of IL-6. DI14 and IL-6 are both Notch-dependent and are required for macrophage polarization through selective down and upregulation of M2- and M1-type markers, respectively. These interesting data underline another relevant aspect the cross-talking between ECs and monocytes/macrophages in evocating inflammation and endothelial dysfunction (see below).

Recently, Foldi and colleagues have also observed that RBP$\mathrm{J}$, a DNA-binding protein that integrates signals from multiple pathways including the Notch pathway, is critically involved in polarization of M2 macrophages. Mice deficient in RBP-J in the myeloid compartment exhibited impaired M2 phenotypes in vivo in a chitin-induced model of M2 polarization. Consistent with the in vivo findings, M2 polarization was partially compromised in vitro in Rbpj-deficient macrophages as demonstrated by reduced expression of a subset of M2 effector molecules including arginase 1. Functionally, myeloid Rbpj deficiency impaired M2 effector functions including recruitment of eosinophils and suppression of $\mathrm{T}$ cell proliferation. Collectively, they have identified RBP-J as an essential regulator of differentiation and function of alternatively activated macrophages (Foldi et al., 2016) (see Fig. 2).

\section{Notch pathway in context of cardiovascular inflammatory conditions related to onset of CVDs, and other age-related diseases}

As discussed in the introductory s' section, endothelium dysfunction is the first pathological condition associated not only with all CVDs, but also with other age-related diseases, being the ECs components of the stroma of all tissues and organs. Thus, in this section, we will stress the role of Notch pathway in the context of cardiovascular inflammatory conditions. On the other hand, the observations, above reported, seem to show a central role of this pathway in their evocation. Furthermore, recent data suggest that it actively participates in dynamic communication and cellular cooperation between innate immunity/inflammatory system, vascular and cardiac cells during both health and pathological conditions. Thus, Notch pathway is not only involved in blood vessel formation, but also in the onset of inflammatory age-related diseases, such as CVDs. This is confirmed by the expression, in vascular and cardiac cells, of all Notch family members, including ligands (Rizzo et al., 2015) (see Fig. 3 A). Here, they are expressed at the basal level with a specific expression pattern in ECs, vascular smooth muscle cells (VSMCs) and fibroblasts. Notch members are also constitutively expressed, as above described, on macrophages and DCs, having a well recognized role in both vascular function and inflammation responses. Expression of Notch molecules correlates with a constitutive activation of Notch pathway (as described above), as reflected by effector hes/hey gene expression and CBF-1 reporter assays (Ando et al.,2003).

In case of age-related inflammatory CVDs, a key role of Notch pathway has been also underlined (see Fig. 3B). An example is given by large-vessel vasculitis. In this pathological condition, it has been demonstrated that Notch pathway blocks Jag1-Fc or acts through the activation of a $\gamma$-secretase inhibitor. This determines immunosuppressive effects, including reduction of T-cell infiltration and proliferation. As result, Th1 and Th17 responses are attenuated through the reduction of IFN $\gamma$ and IL17 production in tissue, respectively (Piggot et al., 2011). In addition, it has been also observed that Notch pathway has a role in limiting inflammatory condition, by inducing the release of IL-10 by T-cells via Dll4 presentation on plasmacytoid dendritic cells (pDCs) (Kassner et al., 2010).

Other studies have suggested a crucial role of Notch pathway during atherosclerosis evocation. In particular, Outtz and colleagues recently evidenced, that Notch-1 partial deletion (Notch1+/- hemizygous mice) induces a decrease in the recruitment of macrophages at the site of vascular injury associated with an impaired production of TNF- $\alpha$ in the wound, when compared to wild-type controls. Macrophages isolated from Notch-1+/- individuals produce and release reduced levels of pro-inflammatory cytokines (TNF, IL6, IL12) and chemokines (CXCL10, CCL2) in response to IFN $\gamma$, when compared with Notch-1+/+ derived cells (Outtz et al., 2010). By determining constitutive activation of Notch1 in macrophages, it has been also observed an increased induction of IRF-1 (interferon regulatory factor 1), SOCS1 (suppressor of cytokine signaling-1), ICAM1 (intercellular adhesion molecule-1) and MHC-II (major histocompatibility complex, class II) by IFN $\gamma$, and a reduction of NO (nitric oxide) production (Monsalve et al., 2006). Interestingly, it has been also observed that Dll4 reduces the production of the IL8 chemokine in response to ischemia or TNF$\alpha$, and selectively induces the expression of inflammatory genes, such as iNOS, pentraxin 3 and Id1 (Fung et al., 2007; Al Haj Zen et al., 2010).

Thus, the data of these studies suggest that Notch pathway in macrophages seems to participate and favor pro-inflammatory responses by releasing more cytokines and chemokines, likely through Notch-1 and Notch-3 activation. Co-temporally, they also evidence that in EC cells, Notch-4, (the typical Notch pathway of EC cells and not of macrophages) evocates anti-inflammatory functions, by reducing the TNF-mediated induction of vascular cell adhesion molecule-1 (VCAM-1), involved in the recruitment of immune cells to inflammatory sites (Quillard et al., 2010). Recently, Briot and cowokers also proposed that Notch-1 can function as an antagonist of EC activation. In particular, they have observed that Notch-1 was constitutively expressed by adult arterial endothelium, but its levels significantly decreased in highfat diet condition. Furthermore, treatment of human aortic ECs (HAECs) with inflammatory lipids and proinflammatory cytokines (TNF and IL1 $\beta$ ) reduced Notch-1 expression and signaling in vitro through a mechanism, requiring STAT3 activation. In addition, the decrease of Notch-1 in HAECs induced by siRNA action, and in the absence of inflammatory lipids or cytokines, augmented inflammatory molecules and the binding of monocytes. Interestingly, the reduction of Notch-1 was also associated with a genetic 


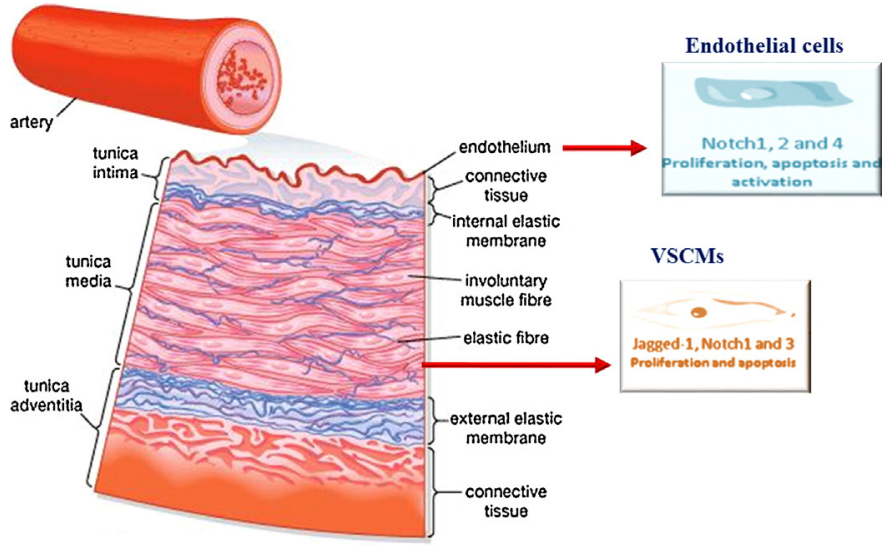

(A)

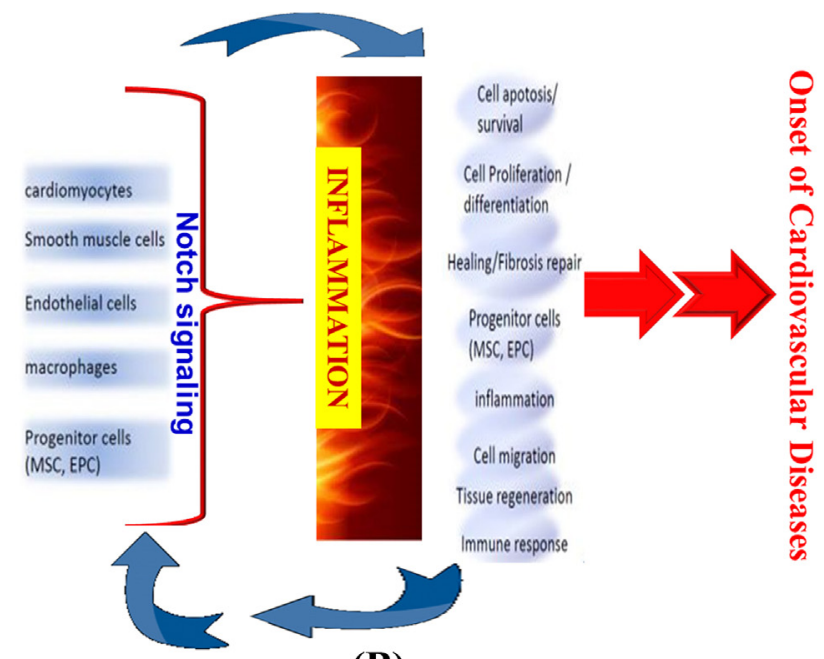

(B)

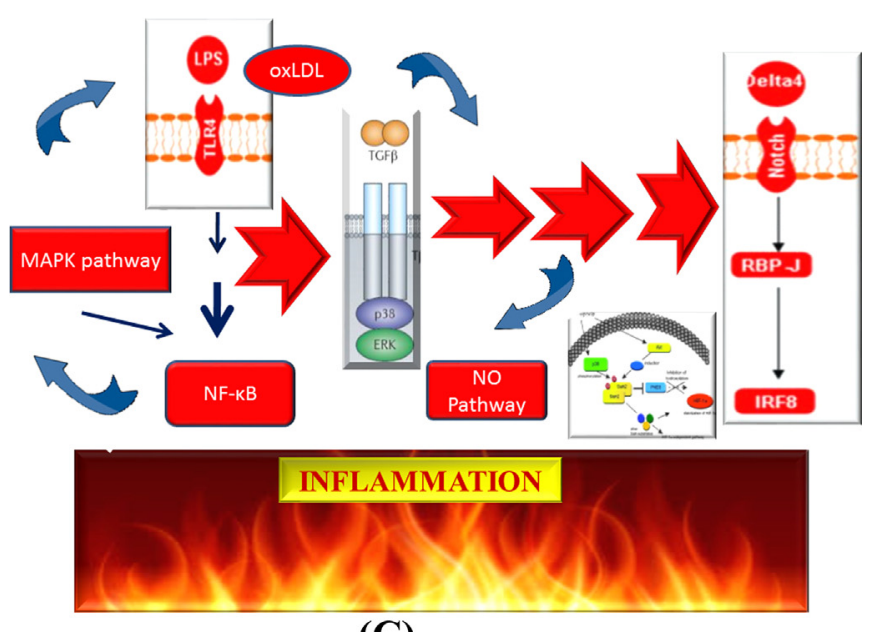

(C)

Fig. 3. $(A-C)$ : in $(A)$, Notch members in the cells of vascular wall. Notch members are expressed on all cardiovascular cells. In particular, Notch 1, 2, and 4 are expressed on EC cells, by modulating their proliferation, apoptosis and activation in a precise microenvironment context. On VSMCs, Notch 1 and 3 are expressed, by controlling their proliferation and apoptosis. In (B), Notch pathway in cardiovascular inflammatory conditions. Notch pathway expressed on cardiovascular and inflammatory/innate cells in inflammatory conditions has several implications on these cells, altering cell activation, recruitment, differentiation, proliferation, migration and/or apoptosis, tissue repair and regeneration. In addition, it modulates the dynamic communication and cellular cooperation between innate immunity/inflammatory cells and vascular and cardiac cells. This determines of a serial of events which characterizes the onset of inflammatory CVDs, including atherosclerosis. In (C), Cross-talks between Notch and inflammatory variant previously correlated to high-density lipoprotein in a human genome-wide association study. Based on these findings, they proposed that reduction of endothelial Notch-1 is a predisposing factor in the onset of vascular inflammation and initiation of atherosclerosis (Briot et al., 2015).

Furthermore, it has been described that during onset of chronic age-related inflammatory CVDs (i.e. atherosclerosis and its complications, such as MI), Notch pathway is also involved in controlling the phenotypic changes of cardiovascular cells. Accordingly, there is a typical accumulation of VSMC/mesenchymal-like cells in these pathological conditions, which exhibit low contractility and high capacity to proliferate and migrate from the media to the neointima. These cells are derived from resident cells and/or circulating progenitor cells. The Notch pathway is strongly involved in cell fate control and, therefore, stands as a potentially important player in pathological remodeling processes (Fiúza and Arias, 2007). Notch controls both the differentiation and the phenotypes of vascular cells. In particular, in deregulated EC cells the activation of Notch-4 determine loss of endothelial markers (i.e. vascular endothelialcadherin, Tie1, Tie2, platelet-endothelial cell adhesion molecule-1 and endothelial NO synthase), and the expression of mesenchymal markers (alpha-smooth muscle actin, fibronectin and plateletderived growth factor receptors), followed by migration toward platelet-derived growth factor-BB. Similarly, Jag1 stimulation also triggers this endothelial-to-mesenchymal transformation (EMT) (Noseda et al., 2004a). In VSMC cells, Notch-1 and Notch-3 receptors are key regulators of their differentiation (Rizzo et al., 2015). Furthermore, in response to inflammatory stimuli, such as IL1 $\beta$, the VSMCs change phenotype towards an inflammatory or dedifferentiated state, which occurs notably through down-regulation of VSMC markers and actin cytoskeleton reorganization. It has been shown that activation of the Notch pathway by Dll1 or by the active form of Notch-3 avoids this phenomenon, while Notch pathway blockade by a $\gamma$-secretase inhibitor improves it (Clémente et al., 2007).

In addition, relevant data evidenced that during these pathological conditions, Notch pathway also affects EC and VSMC cell proliferation and migration and their key cellular functions associated with the onset of vascular remodeling. In vascular physiological conditions, Notch pathway controls vascular cell quiescence through a basal expression of Notch receptors and ligands in ECs and VSMCs. This consents that only $0.01 \%$ of cells are actively proliferating (Rizzo et al., 2015). As contacts between cells increase when confluence is reached, the Notch signaling pathway would therefore stand as a molecular mechano-sensor for quiescence control by inhibiting vascular cells proliferation. Other research groups demonstrated that when ECs reach confluence, Notch signaling activity augments, while $\mathrm{p} 21 \mathrm{Cip} 1$ is concomitantly down-regulated

signaling pathways. The crucial role of Notch pathway on cell biology of cardiovascular cells in pathological conditions (but also in health status), such as onset of inflammatory CVDs, is the result of a very complex signaling network between Notch itself pathway and other major inflammatory pathways, including TLR, NF$\kappa B$, MAPK, TGF- $\beta$, NO and hypoxia pathways. They show multifaceted interactions at multiple levels. In particular, activation of TLR-4 pathway by linking PAMP or DAMP ligands (i.e. LPS and oxLDL, respectively) on EC cells determines activation of NF-kB and MAPK pathways and cotemporally increased expression of Notch receptors and ligands, which result in alterations culminating in the evocation of macrophage infiltration and activation also mediated by a crosstalk between TLR4- NF-kB and Notch pathways. This consequently induces release of inflammatory mediatory which active stress and stretch pathways expressed on ECs, VSMCs and fibroblasts, such as NO pathway and TGF- $\beta$ pathways. Such activation of TGF- $\beta$ pathway determines release of metalloproteinases, tissue injury and consequent DAMP release. As vicious circle, these last additionally active and increase the expression of TLR-4- NF-kB and Notch pathways. As ulterior result, they induce a decrease in the level of oxygen and a cellular hypoxic response. All these events converge in pathological changes, including endothelial dysfunction and vascular remodeling, which are the causes of onset of inflammatory CVDs, i.e. atherosclerosis. 
(Noseda et al., 2004b; Emuss et al., 2009). Inhibition of Notch pathway at confluence prevents p21Cip1 downregulation and induces $\mathrm{Rb}$ phosphorylation and proliferation. In VSMCs, it has been also demonstrated that proper regulation of Notch3 activation is required at confluence to up-regulate cell cycle inhibitor p27kip expression and, subsequently, block cell growth (Campos et al., 2002). Notch signaling alters VSMC growth, but in a precise manner as Notch-1 and Notch-3 activation, significantly increased VSMC proliferation (Sweeney et al., 2004). Moreover, primary VSMCs from hey2 $-1-$ mice proliferate at a reduced rate compared with wild-type cells, whereas the over-expression of hey 1 in VSMCs leads to increased proliferation associated with reduced levels of the cyclin-dependent kinase inhibitors. p21waf1/cip1 (Cdkn1a) and p27kip1 (Cdkn1b). In this latter study, the authors demonstrated that hey2 repressor directly interacts with the p27kip1 promoter to block its transcription (Sakata et al., 2004).

In addition to Notch influence on cell phenotype, growth and migration of cardiovascular cells, it has been observed during agerelated inflammatory CVDs, Notch pathway also affects EC survival. Precisely, the Notch-4 activation level regulates EC survival and protects from apoptosis (MacKenzie et al., 2004). Endothelial apoptosis protection can be mediated through a CBF-1-independent mechanism that triggers the induction of Bcl-2 expression. In contrast, loss of Notch-4 and Hes1 signaling by siRNA in experimental studies elicits apoptosis (Quillard et al., 2008). Furthermore, it has been likely observed that the Notch expression pattern modulation induced by inflammatory cytokines, such as TNF- $\alpha$, characterized by a decrease in Notch- 4 expression and an increase in Notch2, can act as an important event that leads to endothelial dysfunctionassociated apoptosis (Quillard et al., 2010). In VSMCs, it has been found that Notch-1 and Notch-3 activation promotes resistance to apoptosis, which is a prominent feature of the response to injury and regulates the consequent formation of the neointima (Wang et al., 2002). Inhibition of CBF-1 activity with RPMS-1 results in a significant increase in apoptosis following serum deprivation, whereas constitutive expression of Notch-3 and Notch1 ICDs protects VSMCs from death (Campos et al., 2002). Moreover, both transient and constitutive Hey2 over-expression promotes VSMC survival in response to serum deprivation or Fas ligand, in part through the induction of Akt (Wang et al., 2003).

Emerging discoveries have also evidenced a key role of Notch signaling in stroke condition, particularly mediated by Notch-1. On the other hand, it is well recognized that Notch-1 signaling plays a crucial role in all phases of brain development; it regulates the proliferation and differentiation of neural stem cells, and influences cell survival and synaptic plasticity. In addition, there is increasing evidence that Notch pathways are important in neuropathological events, including inflammatory central nervous system disease, brain and spinal cord trauma, and ischemic stroke (Cheng et al., 2014a, 2014b). In particular, it has been reported that the activity of $\gamma$-secretase, and the resulting Notch activation, are elevated in cultured primary cortical neurons subjected to ischemia-like conditions consisting of 3-6h of oxygen and glucose deprivation (Cheng et al., 2014a, 2014b). Moreover, it has been also found that $\gamma$-secretase activity is elevated in ischemic brain tissue in a mouse model of focal ischemic stroke (Cheng et al., 2014a, 2014b). It has been also shown that Notch signaling may interact with hypoxia inducible factor-1 (HIF-1) to contribute to injury outcome following hypoxic conditions (Cheng et al., 2014a, 2014b). In 2015, the group of Baik has also provided the strong evidence that the cooperation between Pin1 (an enzyme that interacts with many cell membrane receptors and signaling proteins that are closely associated with ischemia induced neuronal cell death, such as those within the JNK, p53, and Notch1 signaling pathways) and Notch-1 has a compromising effect on neuronal viability after ischemic stroke, and that the neuronal damage can be inhibited under in vitro and in vivo conditions by treatment with a Pin1 inhibitor (Baik et al., 2015)

Furthermore, it has been also suggested the role of Notch pathway in aneurysm, and particularly in both thoracic and aorta abdominal aneurysm (TAA and AAA). Precisely, the group of Hans demonstrated that, a Notch-1 haplo-insufficiency or pharmacologic inhibition prior to the development of aneurysm dilation at days -7 and +3 of angiotensin II (Ang-II) infusion, dramatically reduces the development of AAA in mice by preventing infiltration of macrophages at the site of vascular injury. This is associated with decreased expression of cytokines and chemokines. Furthermore, they sought to determine the effect of pharmacological inhibition of Notch signaling (DAPT [ $N$-( $N$ - [3,5-difluoro-phenacetyl]-L-alanyl)$S$-phenylglycine t-butyl ester]) on the progression of small AAA after the induction of dilation of the abdominal aorta. Their data showed that pharmacological inhibition of Notch signaling in small AAA attenuates its progression, by not only reducing the inflammatory response, but also increasing differentiation of M2-phenotype macrophages by a Tgf $\beta 2$-dependent mechanism. The abdominal aorta of Apoe $^{-1-}$ mice treated with DAPT also demonstrated increased contents of elastin precursors and adventitial collagen. Thus, these findings suggest that targeting Notch signaling is a promising strategy for reducing AAA progression, particularly in those cases, in which the threshold for surgical intervention for AAA has not yet been reached (Cheng et al., 2014a, 2014b).

In TAA case, the group of LeMaire also underlined a key role of Notch-1, by analysing descending thoracic aortic tissues from patients with sporadic thoracic aortic aneurysm (TAA; $n=14$ ) or chronic thoracic aortic dissection (TAD; $n=16)$ and from agematched organ donors $(n=12)$. Western blots and RT-PCR showed higher levels of the Notch-1 protein and mRNA, and the NICD and Hes1 proteins in both TAA and TAD tissues than in control tissue. However, immune-fluorescence staining showed a complex pattern of Notch signaling in the diseased tissue. The ligand DLL1/4 and Notch-1 were significantly decreased, and NICD and Hes 1 were rarely detected in VSMCs in both TAA and TAD tissues, indicating down-regulation of Notch signaling in aortic VSMCs. Interestingly, Jagged1, NICD, and Hes1 were highly present in CD34+ stem cells and Stro-1+ stem cells in aortas from TAA and TAD patients. NICD and Hes 1 were also detected in most fibroblasts and macrophages that accumulated in the aortic wall of DTAAD patients (Zou et al., 2012)

Based on these relevant discoveries and advances, it is possible to evidence the crucial role of Notch pathway on cell biology of cardiovascular cells in both physiological or pathological conditions. It is the result of a very complex signaling network between Notch itself pathway and other major inflammatory pathways, including TLR, NF- $\kappa B$, MAPK, TGF- $\beta$, NO and hypoxia pathways, which have been in part described above (Fig. 3C).

\section{Conclusions and perspectives}

The Notch signaling is remarkably simple, with just the trimmed receptor that exerts its function in the nucleus, without intervention of classical second messengers. Nevertheless, this highly conserved pathway integrates several additional inputs from other signaling pathways, both in physiological and pathological conditions, such as age-related inflammatory diseases. In this report, we reviewed several examples of such cross-regulation, by describing the potential mechanisms involved. In particular, we evidenced that a very complex signaling network between Notch itself pathway and other major inflammatory pathways, including TLR, NF- $\kappa B$, MAPK, TGF- $\beta$, NO and hypoxia pathways, is associated with onset of inflammatory age-related diseases. The identification of the key regulatory nodes between pathways might represent an 
important base to design more specific inhibitors of a particular pathway, avoiding cross-regulatory side-effects or to use combinatorial treatments. In addition, this knowledge is also forming the base for systems-biology type approaches to predict possible outcomes of receptor stimulation in a quantitative manner. In addition, precise biophysical measurements in the dynamic switch-on and switch-off systems should be used to identify the key regulatory mechanisms. Efforts and progresses in these investigations might give important insights for retarding or delaying age-related diseases, such as CVDs, facilitating their early diagnosis and outcomes. Cooperation among patients, biochemists, physicians, researchers, is essential in supporting this common goal. Obstacles might be many, and the road hard and long, but the advances obtained might be different and offer us greater and unique opportunities to meet the imposing challenge.

\section{Conflict of interests}

The authors declare that they have no conflict of interests.

\section{Authors' Contribution}

Dr Balistreri firstly contributed to conception and design, and in drafting the major number of the sections of paper. Dr Madonna was involved in drafting the 2 and 3 sections. Drs Balistreri and Madonna and Profs. Caruso and Melino contributed to the critical revision. All authors participated in the study, and they read and approved the final version of the paper.

\section{Acknowledgments}

This work has been supported by grants from the Italian Ministry of Education, University and Research to Dr Balistreri CR and Prof G. Mazzesi (000134_RDB_2014 "Focus on cellular and molecular mechanisms of both, protection and repair in BAV vs. TAV patients as novel tools for personalized treatments").

\section{References}

Aguilera, C., Hoya-Arias, R., Haegeman, G., Espinosa, L., Bigas, A., 2004. Recruitment of IkappaBalpha to the hes1 promoter is associated with transcriptional repression. Proc. Natl. Acad. Sci. U. S. A. 101, 16537-16542.

Al Haj Zen, A., Oikawa, A., Bazan-Peregrino, M., Meloni, M., Emanueli, C., Madeddu, P., 2010. Inhibition of delta-like-4-mediated signaling impairs reparative angiogenesis after ischemia. Circ. Res. 107 (2), 283-293.

Alberi, L., Hoey, S.E., Brai, E., Scotti, A.L., Marathe, S., 2013. Notch signaling in the brain: in good and bad times. Ageing Res. Rev. 12 (3), 801-814.

Amsen, D., Blander, J.M., Lee, G.R., Tanigaki, K., Honjo, T., Flavell, R.A., 2004. Instruction of distinct CD4 T helper cell fates by different notch ligands on antigen-presenting cells. Cell 117 (4), 515-526.

Amsen, D., Helbig, C., Backer, R.A., 2015. Notch in t cell differentiation: all things considered. Trends Immunol. 36 (12), 802-814.

Ando, K., Kanazawa, S., Tetsuka, T., Ohta, S., Jiang, X., et al., 2003. Induction of Notch signaling by tumor necrosis factor in rheumatoid synovial fibroblasts. Oncogene 22, 7796-7803.

Aquila, G., Pannella, M., Morelli, M.B., Caliceti, C., Fortini, C., Rizzo, P., Ferrari, R., 2013. The role of Notch pathway in cardiovascular diseases. Glob. Cardiol. Sci. Pract. 2013 (4), 364-371.

Baik, S.H., Fane, M., Park, J.H., Cheng, Y.L., Yang-Wei Fann, D., Yun, U.J., Choi, Y., Park, J.S., Chai, B.H., Park, J.S., Back, S.H., Jeong, J.I., Jang, Y.J., Bahn, G., Lee, J.Y., Li, Y.I., Sobey, C.G., Uchida, T., Park, J.H., Kim, H.T., Tang, S.C., Arumugam, T.V., Jo, D.G. 2015. Pin 1 promotes neuronal death in stroke by stabilizing Notch intracellular domain. Ann. Neurol. 77 (3), 504-516.

Balint, K., Xiao, M., Pinnix, C.C., Soma, A., Veres, I., Juhasz, I., Brown, E.J., Capobianco, A.J., Herlyn, M., Liu, Z.J., 2005. Activation of Notch1 signaling is required for beta-catenin-mediated human primary melanoma progression. J. Clin. Invest. 115 (11), 3166-3176.

Balistreri, C.R., Colonna-Romano, G., Lio, D., Candore, G., Caruso, C., 2009. TLR-4 polymorphisms and ageing: implications for the pathophysiology of age-related diseases. J. Clin. Immunol. 29 (4), 406-415.

Balistreri, C.R., Pisano, C., Merlo, D., Fattouch, K., Caruso, M., Incalcaterra, E., ColonnaRomano, G., Candore, G., 2012. Is the mean blood leukocyte telomere length a predictor for sporadic thoracic aortic aneurysm? Data from a preliminary study. Rejuvenation Res. 15 (2), 170-173.
Balistreri, C.R., Candore, G., Accardi, G., Colonna-Romano, G., Lio, D., 2013. NF-кВ pathway activators as potential ageing biomarkers: targets for new therapeutic strategies. Immun. Ageing 10 (1), 24.

Balistreri, C.R., Pisano, C., Martorana, A., Triolo, O.F., Lio, D., Candore, G., Ruvolo, G., 2014. Are the leukocyte telomere length attrition and telomerase activity alteration potential predictor biomarkers for sporadic TAA in aged individuals? Age (Dordr) 36 (5), 9700.

Balistreri, C.R., 2015. Genetic contribution in sporadic thoracic aortic aneurysm? Emerging evidence of genetic variants related to TLR-4-mediated signaling pathway as risk determinants. Vascul. Pharmacol. 74, 1-10.

Barker, D.J., Osmond, C., Golding, J., Kuh, D., Wadsworth, M.E., 1989. Growth in utero, blood pressure in childhood and adult life, and mortality from cardiovascular disease. BMJ 298 (6673), 564-567.

Barker, D.J.P., Osmond, C., Kajantie, E., Eriksson, J., 2009. Growth and chronic disease: findings in the Helsinki Birth Cohort. Ann. Hum. Biol. 36, 445-458.

Barker, D.J., 2004. The developmental origins of adult disease. J. Am. Coll. Nutr. 23, 588595S.

Bigas, A., Espinosa, L., 2012. Hematopoietic stem cells: to be or Notch to be. Blood 119 (14), 3226-3235.

Bigas, A., Martin, D.I., Milner, L.A., 1998. Notch1 and Notch2 inhibit myeloid differentiation in response to different cytokines. Mol. Cell. Biol. 18 (4), 2324-2333.

Bodnar, A.G., Ouellette, M., Frolkis, M., Holt, S.E., Chiu, C.P., Morin, G.B., Harley, C.B. Shay, J.W., Lichtsteiner, S., Wright, W.E., 1998. Extension of life-span by introduction of telomerase into normal human cells. Science 279 (5349), 349-352.

Borggrefe, T., Oswald, F., 2009. The Notch signaling pathway: transcriptional regulation at Notch target genes. Cell. Mol. Life Sci. 66 (10), 1631-1646.

Borggrefe, T., Lauth, M., Zwijsen, A., Huylebroeck, D., Oswald, F., Giaimo, B.D., 2016. The Notch intracellular domain integrates signals from Wnt, Hedgehog, TGFß/BMP and hypoxia pathways. Biochim. Biophys. Acta 1863 (2), 303-313.

Borillo, G.A., Mason, M., Quijada, P., Völkers, M., Cottage, C., McGregor, M., Din, S., Fischer, K., Gude, N., Avitabile, D., Barlow, S., Alvarez, R., Truffa, S., Whittaker, R., Glassy, M.S., Gustafsson, A.B., Miyamoto, S., Glembotski, C.C., Gottlieb, R.A Brown, J.H., Sussman, M.A., 2010. Pim-1 kinase protects mitochondrial integrity in cardiomyocytes. Circ. Res. 106 (7), 1265-1274.

Brandt, S., 2010. TERT over-expression affects the growth of myocardial tissue derived from mouse embryonic stem cells. Differentiation 79 (1), 1-8.

Bray, S.J., 2006. Notch signalling: a simple pathway becomes complex. Nat. Rev. Mol. Cell Biol. 7 (9), 678-689.

Briot, A., Civelek, M., Seki, A., Hoi, K., Mack, J.J., Lee, S.D., Kim, J., Hong, C., Yu, J., Fishbein, G.A., Vakili, L., Fogelman, A.M., Fishbein, M.C., Lusis, A.J., Tontonoz, P., Navab, M., Berliner, J.A., Iruela-Arispe, M.L., 2015. Endothelia NOTCH1 is suppressed by circulating lipids and antagonizes inflammation during atherosclerosis. J. Exp. Med. 212 (12), 2147-2163.

Brown, M.S., Ye, J., Rawson, R.B., Goldstein, J.L., 2000. Regulated intramembrane proteolysis: a control mechanism conserved from bacteria to humans. Cell 100 (4), 391-398.

Calkins, K., Devaskar, S.U., 2011. Fetal origins of adult disease. Curr. Probl. Pediatr. Adolesc. Health Care 41 (6), 158-176.

Campisi, J., Andersen, J.K., Kapahi, P., Melov, S., 2011. Cellular senescence: a link between cancer and age-related degenerative disease. Semin. Cancer Biol. 21, 354-359.

Campos, A.H., Wang, W., Pollman, M.J., Gibbons, G.H., 2002. Determinants of Notch-3 receptor expression and signaling in vascular smooth muscle cells: implications in cell-cycle regulation. Circ. Res. 91 (11), 999-1006.

Candi, E., Rufini, A., Terrinoni, A., Giamboi-Miraglia, A., Lena, A.M., Mantovani, R., Knight, R., Melino, G., 2007. DeltaNp63 regulates thymic development through enhanced expression of FgfR2 and Jag2. Proc. Natl. Acad. Sci. U. S. A. 104 (29) 11999-12004.

Caolo, V., Schulten, H.M., Zhuang, Z.W., Murakami, M., Wagenaar, A., Verbruggen, S., Molin, D.G., Post, M.J., 2011. Soluble Jagged-1 inhibits neointima formation by attenuating Notch-Herp2 signaling. Arterioscler. Thromb. Vasc. Biol. 31 (5), 1059-1065

Carlson, M.E., Silva, H.S., Conboy, I.M., 2008a. Aging of signal transduction pathways, and pathology. Exp. Cell Res. 314 (9), 1951-1961.

Carlson, M.E., Hsu, M., Conboy, I.M., 2008b. Imbalance between pSmad3 and Notch induces CDK inhibitors in old muscle stem cells. Nature 454 (7203), 528-532.

Carlson, M.E., Suetta, C., Conboy, M.J., Aagaard, P., Mackey, A., Kjaer, M., Conboy, I., 2009. Molecular aging and rejuvenation of human muscle stem cells. EMBO Mol. Med. 1 (8-9), 381-391.

Charville, G.W., Rando, T.A., 2011. Stem cell ageing and non-random chromosome segregation. Philos. Trans. R. Soc. Lond. B Biol. Sci. 366, 85-93.

Cheng, J., Koenig, S.N., Kuivaniemi, H.S., Garg, V., Hans, C.P., 2014a. Pharmacologica inhibitor of notch signaling stabilizes the progression of small abdominal aortic aneurysm in a mouse model. J. Am. Heart Assoc. 3 (6), e001064, http://dx.doi. org/10.1161/JAHA.114.001064.

Cheng, Y.L., Park, J.S., Manzanero, S., Choi, Y., Baik, S.H., Okun, E., Gelderblom, M., Fann, D.Y., Magnus, T., Launikonis, B.S., Mattson, M.P., Sobey, C.G., Jo, D.G., Arumugam, T.V., 2014b. Evidence that collaboration between HIF-1 $\alpha$ and Notch-1 promotes neuronal cell death in ischemic stroke. Neurobiol. Dis. 62, 286-295.

Choudhery, M.S., Khan, M., Mahmood, R., Mohsin, S., Akhtar, S., Ali, F., Khan, S.N. Riazuddin, S., 2012. Mesenchymal stem cells conditioned with glucose depletion augments their ability to repair-infarcted myocardium. J. Cell Mol. Med. 16 (10), 2518-2529.

Chung, H.Y., Cesari, M., Anton, S., Marzetti, E., Giovannini, S., Seo, A.Y., Carter, C., Yu, B.P., Leeuwenburgh, C., 2009. Molecular inflammation: underpinnings of aging and age-related diseases. Ageing Res. Rev. 8 (1), 18-30. 
Clément, N., Gueguen, M., Glorian, M., Blaise, R., Andréani, M., Brou, C., Bausero, P., Limon, I., 2007. Notch3 and IL-1beta exert opposing effects on a vascular smooth muscle cell inflammatory pathway in which NF-kappaB drives crosstalk. J. Cell Sci. 120 (Pt 19), 3352-3361.

Conboy, I.M., Conboy, M.J., Smythe, G.M., Rando, T.A., 2003. Notch-mediated restoration of regenerative potential to aged muscle. Science 302 (5650), 1575-1577.

Conboy, I.M., Conboy, M.J., Wagers, A.J., Girma, E.R., Weissman, I.L., Rando, T.A. 2005. Rejuvenation of aged progenitor cells by exposure to a young systemic environment. Nature 433 (7027), 760-764

Cruickshank, M.N., Ulgiati, D., 2010. The role of notch signaling in the development of a normal B-cell repertoire. Immunol. Cell Biol. 88 (2), 117-124.

D’Souza, B., Miyamoto, A., Weinmaster, G., 2008. The many facets of Notch ligands. Oncogene 27 (38), 5148-5167.

D’Souza, B., Meloty-Kapella, L., Weinmaster, G., 2010. Canonical and non-canonical Notch ligands. Curr. Top. Dev. Biol. 92, 73-129.

Delaney, C., Varnum-Finney, B., Aoyama, K., Brashem-Stein, C., Bernstein, I.D., 2005. Dose-dependent effects of the Notch ligand Delta1 on ex vivo differentiation and in vivo marrow repopulating ability of cord blood cells. Blood 106 (8) 2693-2699.

de Pooter, R.F., Schmitt, T.M., de la Pompa, J.L., Fujiwara, Y., Orkin, S.H., ZúñigaPflücker, J.C., 2006. Notch signaling requires GATA-2 to inhibit myelopoiesis from embryonic stem cells and primary hemopoietic progenitors. J. Immunol. 176 (9), 5267-5275.

Domenga, V., Fardoux, P., Lacombe, P., Monet, M., Maciazek, J., Krebs, L.T., Klonjkowski, B., Berrou, E., Mericskay, M., Li, Z., Tournier-Lasserve, E., Gridley, T., Joutel, A., 2004. Notch3 is required for arterial identity and maturation of vascular smooth muscle cells. Genes Dev. 18 (22), 2730-2735.

Edwards, R.D., 2012. Population aging, the dependency burden, and challenges facing preventive medicine. Prev. Med. 55 (6), 533-534.

Emuss, V., Lagos, D., Pizzey, A., Gratrix, F., Henderson, S.R., Boshoff, C., 2009. KSHV manipulates Notch signaling by DLL4 and JAG1 to alter cell cycle genes in lymphatic endothelia. PLoS Pathog. 5 (10), e1000616.

Espinosa, L., Cathelin, S., D’Altri, T., Trimarchi, T., Statnikov, A., et al., 2010. The Notch/Hes1 pathway sustains NF-kappaB activation through CYLD repression in T cell leukemia. Cancer Cell 18, 268-281.

Fiúza, U.M., Arias, A.M., 2007. Cell and molecular biology of Notch. J. Endocrinol. 194 (3), 459-474.

Fior, R., Henrique, D., 2009. Notch-Off: a perspective on the termination of Notch signalling. Int. J. Dev, Biol. 53 (8-10), 1379-1384.

Fischer, A., Schumacher, N., Maier, M., Sendtner, M., Gessler, M., 2004. The Notch target genes Hey1 and Hey2 are required for embryonic vascular development. Genes Dev. 18 (8), 901-911.

Fischer, K.M., Cottage, C.T., Wu, W., Din, S., Gude, N.A., Avitabile, D., Quijada, P., Collins, B.L., Fransioli, J., Sussman, M.A., 2009. Enhancement of myocardia regeneration through genetic engineering of cardiac progenitor cells expressing Pim-1 kinase. Circulation 120 (21), 2077-2087.

Foldi, J., Chung, A.Y., Xu, H., Zhu, J., Outtz, H.H., Kitajewski, J., Li, Y., Hu, X., Ivashkiv, L.B., 2010. Autoamplification of Notch signaling in macrophages by TLR-induced and RBP-J-dependent induction of Jagged1. J. Immunol. 185 (9), 5023-5031.

Foldi, J., Shang, Y., Zhao, B., Ivashkiv, L.B., Hu, X., 2016. RBP-J is required for M2 macrophage polarization in response to chitin and mediates expression of a subset of M2 genes. Protein Cell 7 (3), 201-209.

Franceschi, C., Bonafè, M., Valensin, S., Olivieri, F., De Luca, M., Ottaviani, E., De Benedictis, G., 2000. Inflamm-aging: an evolutionary perspective on immunosenescence. Ann. N. Y. Acad. Sci. 908, 244-254.

Fukuda, D., Aikawa, E., Swirski, F.K., Novobrantseva, T.I., Kotelianski, V., Gorgun, C.Z Chudnovskiy, A., Yamazaki, H., Croce, K., Weissleder, R., Aster, J.C., Hotamisligil, G.S., Yagita, H., Aikawa, M., 2012. Notch ligand delta-like 4 blockade attenuates atherosclerosis and metabolic disorders. Proc. Natl. Acad. Sci. U. S. A. 109 (27), E1868-77.

Fung, E., Tang, S.M., Canner, J.P., Morishige, K., Arboleda-Velasquez, J.F., Cardoso, A.A., Carlesso, N., Aster, J.C., Aikawa, M., 2007. Delta-like 4 induces notch signaling in macrophages: implications for inflammation. Circulation 115 (23), 2948-2956.

Gale, N.W., Dominguez, M.G., Noguera, I., Pan, L., Hughes, V., Valenzuela, D.M., Murphy, A.J., Adams, N.C., Lin, H.C., Holash, J., Thurston, G., Yancopoulos, G.D., 2004. Haploinsufficiency of delta-like 4 ligand results in embryonic lethality due to major defects in arterial and vascular development. Proc. Natl. Acad. Sci. U. S. A. 101 (45), 15949-15954.

Garg, V., Muth, A.N., Ransom, J.F., Schluterman, M.K., Barnes, R., King, I.N., Grossfeld, P.D., Srivastava, D., 2005. Mutations in NOTCH1 cause aortic valve disease. Nature 437 (7056), 270-274.

Gordon, S., Plüddemann, A., Martinez Estrada, F., 2014. Macrophage heterogeneity in tissues: phenotypic diversity and functions. Immunol. Rev. 262 (1), 36-55.

Hayflick, L., 2007. Entropy explains aging, genetic determinism explains longevity, and undefined terminology explains misunderstanding both. PLoS Genet. 3 e220.

Heidenreich, P.A., Trogdon, J.G., Khavjou, O.A., Butler, J., Dracup, K., Ezekowitz, M.D., Finkelstein, E.A., Hong, Y., Johnston, S.C., Khera, A., Lloyd-Jones, D.M., Nelson, S.A., Nichol, G., Orenstein, D., Wilson, P.W., Woo, Y.J., 2011. American Heart Association Advocacy Coordinating Committee; Stroke Council; Council on Cardiovascular Radiology and Intervention; Council on Clinical Cardiology; Council on Epidemiology and Prevention; Council on Arteriosclerosis; Thrombosis and Vascular Biology; Councilon Cardiopulmonary; Critical Care; Perioperative and Resuscitation; Council onCardiovascular Nursing; Council on the Kidney in Cardiovascular Disease; Council on Cardiovascular Surgery and Anesthesia, and Interdisciplinary Council onQuality of Care and Outcomes Research. Forecast- ing the future of cardiovascular disease in the United States: a policy statement from the American Heart Association. Circulation. 123 (8), 933-44.

Hu, X., Chung, A.Y., Wu, I., Foldi, J., Chen, J., Ji, J.D., Tateya, T., Kang, Y.J., Han, J. Gessler, M., Kageyama, R., Ivashkiv, L.B., 2008. Integrated regulation of Toll-like receptor responses by Notch and interferon-gamma pathways. Immunity 29 (5), 691-703.

Iso, T., Hamamori, Y., Kedes, L., 2003. Notch signaling in vascular development. Arterioscler. Thromb. Vasc. Biol. 23 (4), 543-553.

Jan, H.M., Wei, M.F., Peng, C.L., Lin, S.J., Lai, P.S., Shieh, M.J., 2012. The use of polyethylenimine-DNA to topically deliver hTERT to promote hair growth. Gene Ther. 19 (1), 86-93.

Kassner, N., Krueger, M., Yagita, H., Dzionek, A., Hutloff, A., Kroczek, R., Scheffold, A. Rutz, S., 2010. Cutting edge: plasmacytoid dendritic cells induce IL-10 production in T cells via the Delta-like-4/Notch axis. J. Immunol. 184 (2), 550-554.

Krebs, L.T., Shutter, J.R., Tanigaki, K., Honjo, T., Stark, K.L., Gridley, T., 2004. Haploinsufficient lethality and formation of arteriovenous malformations in Notch pathway mutants. Genes Dev. 18 (20), 2469-2473.

Kuilman, T., Michaloglou, C., Mooi, W.J., Peeper, D.S., 2010. The essence of senescence. Genes Dev. 24, 2463-2479.

Kumano, K., Chiba, S., Shimizu, K., Yamagata, T., Hosoya, N., Saito, T., Takahashi, T., Hamada, Y., Hirai, H., 2001. Notch1 inhibits differentiation of hematopoietic cells by sustaining GATA-2 expression. Blood 98 (12), 3283-3289.

London, A., Benhar, I., Mattapallil, M.J., Mack, M., Caspi, R.R., Schwartz, M., 2013. Functional macrophage heterogeneity in a mouse model of autoimmune central nervous system pathology. J. Immunol. 190 (7), 3570-3578.

MacKenzie, F., Duriez, P., Wong, F., Noseda, M., Karsan, A., 2004. Notch4 inhibits endothelial apoptosis via RBP-Jkappa-dependent and -independent pathways. J. Biol. Chem. 279 (12), 11657-11663.

Madonna, R., Willerson, J.T., Geng, Y.J., 2008. Myocardin a enhances an enhances telomerase activities in adipose tissue mesenchymal cells and embryonic stem cells undergoing cardiovascular myogenic differentiation. Stem Cells 26 (1), 202-211.

Madonna, R., Wu, D., Wassler, M., De Caterina, R., Willerson, J.T., Geng, YJ., 2013. Myocardin-A enhances expression of promyogenic genes without depressing telomerase activity in adipose tissue-derived mesenchymal stem cells. Int. J. Cardiol. 167 (6), 2912-2921.

Madonna, R., Engel, F.B., Davidson, S.M., Ferdinandy, P., Gorbe, A., Sluijter, J.P., Van Laake, L.W., 2015. Stem cell aging and age-related cardiovascular disease: perspectives of treatment by ex-vivo stem cell rejuvenation. Curr. Drug Targets 16 (8), 780-785.

Madonna, R., Balistreri, C.R., Novo, G., 2016a. Cellular and molecular basis of the imbalance between vascular damage and repair in aging and age-related diseases: as biomarkers and targets for new treatments. Mech. Ageing Dev., pii: S0047-6374(16)30026-4. 10.1016/j.mad.2016.03.005 [Epub ahead of print]

Madonna R. Linda, W. Van Laake, Sean M. Davidson, Felix B. Engel, DerekJ. Hausenloy, Sandrine Lecour, Jonathan Leor, Cinzia Perrino, Rainer Schulz, Kirsti Ytrehus, Ulf Landmesser, Christine L. Mummery, Stefan Janssens, James Willerson, Thomas Eschenhagen, Peter Ferdinandy, Joost P.G. Sluijter, Position Paper of the ESC Working Group Cellular Biology of the Heart: Cell-Based Therapies for Myocardial Repair and Regeneration in Ischemic Heart Disease and Heart Failure. Eur. Heart J. 2016 (in press).

Maniati, Bossard, M., Cook, N., Candido, J.B., Emami-Shahri, N., et al., 2011. Crosstalk between the canonical NF-kappaB and Notch signaling pathways inhibits Ppargamma expression and promotes pancreatic cancer progression in mice. J. Clin. Invest. 121, 4685-4699.

Milner, L.A., Bigas, A., Kopan, R., Brashem-Stein, C., Bernstein, I.D., Martin, D.I., 1996 Inhibition of granulocytic differentiation by mNotch1. Proc. Natl. Acad. Sci. U. S. A. 93 (23), 13014-13019.

Mohsin, S., Khan, M., Nguyen, J., Alkatib, M., Siddiqi, S., Hariharan, N., Wallach, K., Monsanto, M., Gude, N., Dembitsky, W., Sussman, M.A., 2013. Rejuvenation of human cardiac progenitor cells with Pim-1 kinase. Circ. Res. 113 (10), 1169-1179

Mohsin, S., Khan, M., Toko, H., Bailey, B., Cottage, C.T., Wallach, K., Nag, D., Lee, A., Siddiqi, S., Lan, F., Fischer, K.M., Gude, N., Quijada, P., Avitabile, D., Truffa, S., Collins, B., Dembitsky, W., Wu, J.C., Sussman, M.A., 2012. Human cardiac progenitor cells engineered with Pim-I kinase enhance myocardial repair. J. Am. Coll. Cardiol. 60 (14), 1278-1287.

Monastirioti, M., Giagtzoglou, N., Koumbanakis, K.A., Zacharioudaki, E., Deligiannaki, M., Wech, I., Almeida, M., Preiss, A., Bray, S., Delidakis, C., 2010. Drosophila Hey is a target of Notch in asymmetric divisions during embryonic and larval neurogenesis. Development 137 (2), 191-201.

Monsalve, E., Perez, M.A., Rubio, A., Ruiz-Hidalgo, M.J., Baladron, V., et al., 2006 Notch-1 up-regulation and signaling following macrophage activation modulates gene expression patterns known to affect antigen-presenting capacity and cytotoxic activity. J. Immunol. 176, 5362-5373.

Monsalve, E., Ruiz-Garcia, A., Baladron, V., Ruiz-Hidalgo, M.J., Sanchez-Solana, B., et al., 2009. Notch1 upregulates LPS-induced macrophage activation by increasing NF-kappaB activity. Eur. J. Immunol. 39, 2556-2570.

Mumm, J.S., Kopan, R., 2000. Notch signaling: from the outside in. Dev. Biol. 228 (2), $151-165$.

Nemir, M., Metrich, M., Plaisance, I., Lepore, M., Cruchet, S., Berthonneche, C., Sarre A., Radtke, F., Pedrazzini, T., 2014. The Notch pathway controls fibrotic and regenerative repair in the adult heart. Eur. Heart J. 35 (32), 2174-2185.

Niessen, K., Karsan, A., 2008. Notch signaling in cardiac development. Circ. Res. 102 (10), 1169-1181.

Noguera-Troise, I., Daly, C., Papadopoulos, N.J., Coetzee, S., Boland, P., Gale, N.W. Lin, H.C., Yancopoulos, G.D., Thurston, G., 2006. Blockade of Dll4 inhibits 
tumour growth by promoting non-productive angiogenesis. Nature 444 (7122), $1032-1037$.

Noseda, M., Chang, L., McLean, G., Grim, J.E., Clurman, B.E., Smith, L.L., Karsan, A., 2004b. Notch activation induces endothelial cell cycle arrest and participates in contact inhibition: role of p21Cip1 repression. Mol. Cell. Biol. 24 (20), 8813-8822.

Noseda, M., McLean, G., Niessen, K., Chang, L., Pollet, I., Montpetit, R., Shahidi, R., Dorovini-Zis, K., Li, L., Beckstead, B., Durand, R.E., Hoodless, P.A., Karsan, A., 2004a. Notch activation results in phenotypic and functional changes consistent with endothelial-to-mesenchymal transformation. Circ. Res. 94 (7), 910-917.

Ntziachristos, P., Lim, J.S., Sage, J., Aifantis, I., 2014. From fly wings to targeted cancer therapies: a centennial for notch signaling. Cancer Cell 25 (3), 318-334.

Osipo, C., Golde, T.E., Osborne, B.A., Miele, L.A., 2008. Off the beaten pathway: the complex cross talk between Notch and NF-kappaB. Lab. Invest. 88, 11-17.

Ottaviani, S., Tahiri, K., Frazier, A., Hassaine, Z.N., Dumontier, M.F., et al., 2010. Hes1, a new target for interleukin 1beta in chondrocytes. Ann. Rheum. Dis. 69, $1488-1494$.

Outtz, H.H., Wu, J.K., Wang, X., Kitajewski, J., 2010. Notch1 deficiency results in decreased inflammation during wound healing and regulates vascular endothelial growth factor receptor-1 and inflammatory cytokine expression in macrophages. J. Immunol. 185 (7), 4363-4373.

Ovadya, Y., Krizhanovsky, V., 2014. Senescent cells: SASPected drivers of age-related pathologies. Biogerontology 15 (6), 627-642.

Pabois, A., Pagie, S., Gérard, N., Laboisse, C., Pattier, S., Hulin, P., Nedellec, S., Toquet, C., Charreau, B., 2016. Notch signaling mediates crosstalk between endothelial cells and macrophages via Dll4 and IL6 in cardiac microvascular inflammation. Biochem. Pharmacol. 104, 95-107.

Padhee, M., Zhang, S., Lie, S., Wang, K.C., Botting, K.J., McMillen, I.C., MacLaughlin, S.M., Morrison, J.L., 2015. The periconceptional environment and cardiovascular disease: does in vitro embryo culture and transfer influence cardiovascular development and health? Nutrients 7 (3), 1378-1425.

Palaga, T., Buranaruk, C., Rengpipat, S., Fauq, A.H., Golde, T.E., et al., 2008. Notch signaling is activated by TLR stimulation and regulates macrophage functions. Eur. J. Immunol. 38, 174-183.

Perdiguero, E.G., Geissmann, F., 2015. The development and maintenance of resident macrophages. Nat. Immunol. 17 (December (1)), 2-8.

Piggott, K., Deng, J., Warrington, K., Younge, B., Kubo, J.T., Desai, M., Goronzy, J.J., Weyand, C.M., 2011. Blocking the NOTCH pathway inhibits vascular inflammation in large-vessel vasculitis. Circulation 123 (3), 309-318.

Pitale, S., Sahasrabuddhe, A., 2011. Fetal origin of vascular aging. Indian J. Endocrinol. Metab. 15 (October (Suppl. 4)), S289-97.

Poellinger, L., Lendahl, U., 2008. Modulating Notch signaling by pathway-intrinsic and pathway-extrinsic mechanisms. Curr. Opin. Genet. Dev. 18 (5), 449-454.

Qu, Y., Duan, Z., Zhao, F., Wei, D., Zhang, J., Tang, B., Li, J., Yang, C., Mu, D., 2011. Telomerase reverse transcriptase upregulation attenuates astrocyte proliferation and promotes neuronal survival in the hypoxic-ischemic rat brain. Stroke 42 (12), 3542-3550.

Quillard, T., Coupel, S., Coulon, F., Fitau, J., Chatelais, M., Cuturi, M.C., Chiffoleau, E., Charreau, B., 2008. Impaired Notch4 activity elicits endothelial cell activation and apoptosis: implication for transplant arteriosclerosis. Arterioscler. Thromb. Vasc. Biol. 28 (12), 2258-2265.

Quillard, T., Devallière, J., Coupel, S., Charreau, B., 2010. Inflammation dysregulates Notch signaling in endothelial cells: implication of Notch2 and Notch4 to endothelial dysfunction. Biochem. Pharmacol. 80 (12), 2032-2041.

Radtke, F., Wilson, A., Stark, G., Bauer, M., van Meerwijk, J., MacDonald, H.R., Aguet, M., 1999. Deficient T cell fate specification in mice with an induced inactivation of Notch1. Immunity 10 (5), 547-558.

Rando, T.A., Chang, H.Y., 2012. Aging, rejuvenation and epigenetic reprogramming: resetting the aging clock. Cell 148 (1-2), 46-57

Rangarajan, A., Talora, C., Okuyama, R., Nicolas, M., Mammucari, C., Oh, H., Aster, J.C., Krishna, S., Metzger, D., Chambon, P., Miele, L., Aguet, M., Radtke, F., Dotto, G.P., 2001. Notch signaling is a direct determinant of keratinocyte growth arrest and entry into differentiation. EMBO J. 20 (13), 3427-3436.

Regina, C., Panatta, E., Candi, E., Melino, G., Amelio, I., Balistreri, C.R., AnnicchiaricoPetruzzelli, M., Di Daniele, N., Ruvolo, G., 2016. Vascular ageing and endothelial cell senescence: molecular mechanisms of physiology and diseases. Mech. Ageing Dev., pii: S0047-6374(16)30059-8. 10.1016/j.mad.2016.05.003 [Epub ahead of print].

Ridgway, J., Zhang, G., Wu, Y., Stawicki, S., Liang, W.C., Chanthery, Y., Kowalski, J., Watts, R.J., Callahan, C., Kasman, I., Singh, M., Chien, M., Tan, C., Hongo, J.A., de Sauvage, F., Plowman, G., Yan, M., 2006. Inhibition of Dll4 signalling inhibits tumour growth by deregulating angiogenesis. Nature 444 (7122), 1083-1087.

Rizzo, P., Mele, D., Caliceti, C., Pannella, M., Fortini, C., Clementz, A.G., Morelli, M.B., Aquila, G., Ameri, P., Ferrari, R., 2015. The role of notch in the cardiovascular system: potential adverse effects of investigational notch inhibitors. Front. Oncol. 4, 384.

Ruvolo, G., Pisano, C., Candore, G., Lio, D., Palmeri, C., Maresi, E., Balistreri, C.R., 2014. Can the TLR-4-mediated signaling pathway be a key inflammatory promoter for sporadic TAA? Mediators Inflamm. 2014, 349476.

Sahlgren, C., Gustafsson, M.V., Jin, S., Poellinger, L., Lendahl, U., 2008. Notch signaling mediates hypoxia-induced tumor cell migration and invasion. Proc. Natl. Acad. Sci. U. S. A. 105 (17), 6392-6397.

Salminen, A., Kaarniranta, K., 2010. Genetics vs. entropy: longevity factors suppress the NF-kappaB-driven entropic aging process. Ageing Res. Rev. 9, 298-314.
Sakata, T., Sakaguchi, H., Tsuda, L., Higashitani, A., Aigaki, T., Matsuno, K., Hayashi S., 2004. Drosophila Nedd4 regulates endocytosis of notch and suppresses its ligand-independent activation. Curr. Biol. 14 (24), 2228-2236.

Shang, Y., Smith, S., Hu, X., 2016. Role of Notch signaling in regulating innate immunity and inflammation in health and disease. Protein Cell 7 (3), 159-174.

Sica, A., Mantovani, A., 2012. Macrophage plasticity and polarization: in vivo veritas J. Clin. Invest. 122 (3), 787-795.

Sinha, M., Jang, Y.C., Oh, J., Khong, D., Wu, E.Y., Manohar, R., Miller, C., Regalado, S.G., Loffredo, F.S., Pancoast, J.R., Hirshman, M.F., Lebowitz, J., Shadrach, J.L., Cerletti, M., Kim, M.J., Serwold, T., Goodyear, L.J., Rosner, B., Lee, R.T., Wagers, A.J., 2014. Restoring systemic GDF11 levels reverses age-related dysfunction in mouse skeletal muscle. Science 344 (6184), 649-652.

Sweeney, C., Morrow, D., Birney, Y.A., Coyle, S., Hennessy, C., Scheller, A., Cummins, P.M., Walls, D., Redmond, E.M., Cahill, P.A., 2004. Notch 1 and 3 receptor signaling modulates vascular smooth muscle cell growth, apoptosis, and migration via a CBF-1/RBP-Jk dependent pathway. FASEB J. 18 (12), 1421-1423.

Talora, C., Campese, A.F., Bellavia, D., Felli, M.P., Vacca, A., Gulino, A., Screpanti, I., 2008. Notch signaling and diseases: an evolutionary journey from a simple beginning to complex outcomes. Biochim. Biophys. Acta 1782 (9), 489-497.

Terrinoni, A., Serra, V., Bruno, E., Strasser, A., Valente, E., Flores, E.R., van Bokhoven, H., Lu, X., Knight, R.A., Melino, G., 2013. Role of p63 and the Notch pathway in cochlea development and sensorineural deafness. Proc. Natl. Acad. Sci. U. S. A. 110 (18), 7300-7305.

Vasto, S., Candore, G., Balistreri, C.R., Caruso, M., Colonna-Romano, G., Grimaldi, M.P., Listi, F., Nuzzo, D., Lio, D., Caruso, C., 2007. Inflammatory networks in ageing, age-related diseases and longevity. Mech. Ageing Dev. 128 (1), 83-91.

Vu Van, D., Beier, K.C., Pietzke, L.J., Al Baz, M.S., Feist, R.K., Gurka, S., Hamelmann, E., Kroczek, R.A., Hutloff, A., 2016. Local T/B cooperation in inflamed tissues is supported by T follicular helper-like cells. Nat. Commun. 7, 10875.

Wang, H., Zang, C., Liu, X.S., Aster, J.C., 2015. The role of Notch receptors in transcriptional regulation. J. Cell. Physiol. 230 (5), 982-988.

Wang, W., Campos, A.H., Prince, C.Z., Mou, Y., Pollman, M.J., 2002. Coordinate Notch3hairy-related transcription factor pathway regulation in response to arterial injury. Mediator role of platelet-derived growth factor and ERK. J. Biol. Chem. 277 (26), 23165-23171.

Wang, W., Prince, C.Z., Hu, X., Pollman, M.J., 2003. HRT1 modulates vascular smooth muscle cell proliferation and apoptosis. Biochem. Biophys. Res. Commun. 308 (3), 596-601.

Weng, A.P., Ferrando, A.A., Lee, W., 4th, Morris J.P, Silverman, L.B., Sanchez-Irizarry, C., Blacklow, S.C., Look, A.T., Aster, J.C., 2004. Activating mutations of NOTCH1 in human T cell acute lymphoblastic leukemia. Science 306 (October (5694)), 269-271.

Weng, A.P., Millholland, J.M., Yashiro-Ohtani, Y., Arcangeli, M.L., Lau, A., Wai, C., Del Bianco, C., Rodriguez, C.G., Sai, H., Tobias, J., Li, Y., Wolfe, M.S., Shachaf, C., Felsher, D., Blacklow, S.C., Pear, W.S., Aster, J.C., 2006. c-Myc is an important direct target of Notch1 in T-cell acute lymphoblastic leukemia/lymphoma. Genes Dev. 20 (15), 2096-2109.

Wong, L.S., Oeseburg, H., de Boer, R.A., van Gilst, W.H., van Veldhuisen, D.J., van der Harst, P., 2009. Telomere biology in cardiovascular disease: the TERC-/- mouse as a model for heart failure and ageing. Cardiovasc. Res. 81 (2), 244-252.

Wu, L., Aster, J.C., Blacklow, S.C., Lake, R., Artavanis-Tsakonas, S., Griffin, J.D., 2000. MAML1, a human homologue of Drosophila mastermind, is a transcriptional co-activator for NOTCH receptors. Nat. Genet. 26 (4), 484-489.

Xue, Y., Gao, X., Lindsell, C.E., Norton, C.R., Chang, B., Hicks, C., Gendron-Maguire, M., Rand, E.B., Weinmaster, G., Gridley, T., 1999. Embryonic lethality and vascular defects in mice lacking the Notch ligand Jagged1. Hum. Mol. Genet. 8 (5), 723-730.

Yan, B., Zemskova, M., Holder, S., Chin, V., Kraft, A., Koskinen, P.J., Lilly, M., 2003. The PIM-2 kinase phosphorylates BAD on serine 112 and reverses BAD-induced cell death. J. Biol. Chem. 278 (46), 45358-45367.

Yang, A., Kaghad, M., Wang, Y., Gillett, E., Fleming, M.D., Dötsch, V., Andrews, N.C., Caput, D., McKeon, F., 1998. p63, a p53 homolog at 3q27-29, encodes multiple products with transactivating, death-inducing, and dominant-negative activities. Mol. Cell 2 (3), 305-316.

Yang, J., Bücker, S., Jungblut, B., Böttger, T., Cinnamon, Y., Tchorz, J., Müller, M., Bettler, B., Harvey, R., Sun, Q.Y., Schneider, A., Braun, T., 2012. Inhibition of Notch2 by Numb/Numblike controls myocardial compaction in the heart. Cardiovasc. Res. $96(2), 276-285$.

You, L.R., Lin, F.J., Lee, C.T., DeMayo, F.J., Tsai, M.J., Tsai, S.Y., 2005. Suppression of Notch signalling by the COUP-TFII transcription factor regulates vein identity. Nature 435 (7038), 98-104.

Zeng, Q., Li, S., Chepeha, D.B., Giordano, T.J., Li, J., Zhang, H., Polverini, P.J., Nor, J., Kitajewski, J., Wang, C.Y., 2005. Crosstalk between tumor and endothelial cells promotes tumor angiogenesis by MAPK activation of Notch signaling. Cancer Cell 8 (1), 13-23.

Zhang, Q., Wang, C., Liu, Z., Liu, X., Han, C., et al., 2012. Notch signal suppresses TLR-triggered inflammatory responses in macrophages by inhibiting ERK1/2mediated NF-kappaB activation. J. Biol. Chem. 287, 6208-6217.

Zhao, C., Guo, H., Li, J., Myint, T., Pittman, W., Yang, L., Zhong, W., Schwartz, R.J., Schwarz, J.J., Singer, H.A., Tallquist, M.D., Wu, M., 2014a. Numb family proteins are essential for cardiac morphogenesis and progenitor differentiation. Development 141 (2), 281-295.

Zhao, L., Borikova, A.L., Ben-Yair, R., Guner-Ataman, B., MacRae, C.A., Lee, R.T., Burns, C.G., Burns, C.E., 2014b. Notch signaling regulates cardiomyocyte proliferation during zebrafish heart regeneration. Proc. Natl. Acad. Sci. U. S. A. 111 (4), 1403-1408. 
Zhou, L., Li, L.W., Yan, Q., Petryniak, B., Man, Y., Su, C., Shim, J., Chervin, S., Lowe, J.B., 2008. Notch-dependent control of myelopoiesis is regulated by fucosylation. Blood 112 (2), 308-319.

Zippo, A., De Robertis, A., Bardelli, M., Galvagni, F., Oliviero, S., 2004. Identification of Flk-1 target genes in vasculogenesis: pim-1 is required for endothelial and mural cell differentiation in vitro. Blood 103 (12), 4536-4544.
Zou, S., Ren, P., Nguyen, M., Coselli, J.S., Shen, Y.H., LeMaire, S.A., 2012. Notch signaling in descending thoracic aortic aneurysm and dissection. PLoS One 7 (12), e52833. 